\title{
La théorie du régime appliquée à l'analyse des résultats expérimentaux concernant les transports de fond.
}

\author{
PAR \\ T. BLENCH (1) \\ $\mathrm{P} T$ \\ B. SG., M.J.C.N., M.A.S.C.I. \\ Bryan ERB \\ M. sc.
}

English text, p. 148

\begin{abstract}
A titre d'introduction, présentation rapide de l'essence, de l'origine et plus particulierement de l'application an calcul des canaux, de la théorie du régime. Les méthodes dérivées de la théorie du régime sont appliquées à une analyse des observations recueillies et publiees sur les transports de sable et de gravitlon dans des canalux expérimentaux en labo-
\end{abstract}

\begin{abstract}
ratoire. Des résultats simples et originanx sont obtenus, la théorie du régime bénéficie d'une certaine généralisation et les résultats exploites s'aberent insuffisants pour coubrir tout le domaine de la pratique. Présentation de suggestions pour une coopérative internalionale de recherches.
\end{abstract}

\section{Introduction}

Il y a deux façons essentielles d'aborder le problème des transports de fond; elles sont diamétralement opposées.

La théorie du régime [1], [2], [3], [10], [11], créée aux Indes à la fin du xix" siècle a fourni des formules que le gouvernement des Indes a admis en 1933 à la base de ses projets [13]. Ces formules cadrent avec l'hydraulique des écoulements limités par des parois rigides al les généralisent même. On y est arrivé par l'étude des relations liant la vitesse à la largeur, à la profondeur et à la pente de ces canaux qui ont fini par se régler à un état d'équilibre (de régime) en dépit des vains efforts de certains ingénieurs pour les obliger à enfreindre les lois de la nature. Par leur origine même, ces for-

(1) Professeur de Génie civil à I'Université d'Alberta (Canada) et ingénicur-conseil.

(2) Ingénieur préposé aux recherches à Ia A.V. Roe $\mathrm{C}^{\circ}$, Toronto (Canada) mules définissent en fonction de $r, b, d$, des coefficients caractérisant les matériaux de fond et l'état de cohésion des parois; elles ignorent les propriétés physiques des matériaux de fond et de paroi qui sont la raison d'être de ces coefficients; par là même elles se rapprochent des équations de l'électro-magnétisme qui définissent des «quantités ; d'électricité et de magnétisme d'après les résultats auxquels elles conduisent, non d'après les causes qui les ont provoquées, imparfaitement élucidées. Mathématiquement parlant, on pourrait dire que les formules de « régime» constituent ce qui resterait des formules de transport solide après en avoir éliminé les paramètres qui caractérisent les sédiments et, évidemment, en supposant ces formules connues.

La méthode expérimentale, comme nous l'appellerons dans ce qui suit, s'est développée en Europe et aux Etats-Unis d'Amérique à la suite d'étude, sur canaux de laboratoire, d'écoule- 
ments à transport solide. En effet, on ne disposait pas là des grands réseaux de canaux autoréglés et régulic̀rement observés nécessaires à une étude directe. Les nombreuses formules mises sur pied s'efforcaient d'établir une relation entre les caractéristiques de l'écoulement discernables sur ces canaux et les caractéristiques mesurables du matériau de fond; d'autres tentatives se rapportaient au transport en suspension qui sort du cadre de cet article. Celles des formules qui, du point de vue dynamique, semblent reposer sur l'assise la meilleure $[7,9]$ revètent aujourd'hui une forme si compliquée que peu d'ingénieurs arrivent à en saisir l'origine et les modalités d'emploi, ou à discerner la gamme des données dont elles découlent. Elles n'ont d'ailleurs pas de rapport aux données premières, mais plutôt à des grandeurs qu'on en fait dériver dans l'hypothèse d'un écoulement uniforme sur une portion du canal et en en déduisant la part de la section qui pourrait « être attribuée au lit» si l'on voulait appliquer la formule de Manning.

Les auteurs estiment qu'en principe les deux facons de procéder sont légitimes et qu'elles devraient être développées jusqu'à ce qu'elles concordent. Ils ont cherché à y apporter leur contribution en utilisant les méthodes de la théorie du régime à l'analyse des résultats classiques de Grbert [4], lesquels semblent constituer le noyau des travaux de Meyer-PeTer et Enstern [7] [9], ainsi que des observations recueillies plus récemment en laboratoire sur le transport en canaux de sables naturels de rivière [16] [17]. Ce fut un gros travail, mais les méthodes sur lesquelles il repose sont extrêmement simples et les résultats auxquels on arrive faciles à saisir. Son plus grand mérite réside peut-être dans la constatation que les données expérimentales actuelles sont tout à fait insuffisantes pour établir des formules pratiquement utilisables pour les projets; la voie est donc tracée à de nouvelles recherches.

Les auteurs exposent dans ce qui suit leur façon de voir et les résultats auxquels ils sont parvenus. Disposant de peu d'espace, ils n'en peuvent donner qu'un apercu, les ouvrages cités a la fin de l'article pouvant fournir au chercheur les détails dont il manquerait. Etant donnć le caractère fondamental de la théorie du régime, les trois chapitres qui suivent lui sont consacrés; ils reproduisent à peu près textuellement l'ouvrage réf. 10 , avec l'autorisation de l'A.S.C.E. Le lecteur familier avec d'autres écrits sur la question ne manquera pas de noter que nous avons modifié les notations pour nous conformer aux recommandations de l'A.S.C.E.; il notera que la formule donnant la pente contient déjà en germe un des résultats auxquels l'article conduit.

\section{NOTATIONS}

Sauf indication contraire, tontes les unites

sont rapportées au système pieds-secondes

$b=$ Largeur du chenal;

$C=$ Charge, c'est-à-dire rapport entre le poids du sédiment passant en une seconde divisée par le poids de l'eau passant en une seconde, exprimé en millièmes de pourcent;

$\mathrm{C}_{\mathrm{c}}=$ Charge à la vitesse critique;

$\mathrm{K}=$ Aspect généralisé de la constante non dimensionnelle entrant dans l'équation de King;

$d=$ Profondeur de l'écoulement;

$\mathrm{D}=$ Diamètre des particules formant le fond;

$\mathrm{D}_{m}=$ Diamètre mesuré en $\mathrm{mm}$;

$\mathrm{F}_{b}=$ Coefficient de fond $\mathrm{V}^{2} / d$;

$\mathrm{F}_{l 0}=$ Coefficient de fond, quand $\mathrm{C}$ devient infiniment petit;

$g=$ Accélération de la pesanteur;

$v=$ Viscosité cinématiqne;

$a=$ Constante;

$\mathrm{S}=$ Pente du chenal quand la pente de la surface de l'eau est supposée la même;

$\mathrm{V}=$ Vitesse moyenne de l'écoulement;

$x=$ Constante;

Comme chez Grubert et Murphy, les initiales $\mathrm{A} \dot{a} \mathrm{H}$ indiquent les variétés de sables utilisées.

$f n=$ signifie « fonction non spécifiée de

$\mathrm{K}=\left(\mathrm{V}^{2} / g d \mathrm{~S}\right) \div(\mathrm{Vb} / \mathrm{y})^{0,25}$

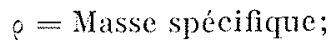

$\tau=$ Intensité du frottement tangentiel.

\section{Les conditions générales de validité des équations de la théorie du régime}

Les équations de la théorie du régime ont été établies conformément aux lois de la dynamique, pour traduire les corrélations observées entre les paramètres mesurables caractérisant les canaux artificiels dont le fond non cohérent se déplace par dunes, dont les parois sont meubles et cohérentes, dont la largeur, la profondeur et la pente se riglent finalement à des valeurs constituant ce que l'on appelle l'état de régime. On en conclura que ces équations sont rigoureusement valables pour :

1. Les canaux rectilignes;

2. Dont les parois se comportent comme si elles étaient « lisses », hydrauliquement parlant (en langage technique, cela veut dire que le coefficient de rugosité des parois ne dépend que de la nature du fluide, aussi complexe qu'elle soit);

3. Dont le lit est au moins trois fois plus large que profond; 
4. Dont les parois ont une pente à peu près égale à celle des talus naturels;

5. Qui ont un débit constant;

6. Dont la charge en matériaux est invariable, aussi complexe soit-elle;

7. Dont le fond non cohérent se déplace, même très lentement, sous forme de dune;

8. Dont la vitesse est inférieure à la vitesse critique;

9. Dont la largeur, la profondeur et la pente ont atteint leurs valeurs finales (on de régime). (On suppose qu'en dehors de certains cas tres peu probables, un canal comme celui qui est considéré ici n'est pas destiné à véhiculer sa charge solide et son débit liquide sans engraisser ou éroder ses parois; il y aura done adaptation automatique jusqu'à formation de l'état de régime.)

\section{Les conditions pratiques de validité} des équations de la théorie du régime

L'expérience et l'observation attentive des principes de la dynamique permettent d'étendre les équations de la théorie du régime bien au-delà de ces limites théoriques de validité, lors même qu'il s'agirait de rivières très chargées ou d'estuaires à marée [5], [6], [10]. En ce qui concerne les cours d'eau aménagés pouvant être raisonnablement rangés parmi les canaux, les réflexions ci-après, se rapportant aux différents points que nous avons énumérés au chapitre précédent, montrent jusqu'où peut aller la tolérance dans l'application des équations à la majorité des problèmes de la pratique:

1. Ón pourra négliger la courbure dans les cours d'eau ordinaires. A l'extérieur de la courbe, la section s'approfondira, à l'intérieur le fond tendra à se relever. Mais on n'a pas l'habitude de procéder à des terrassements pour devancer cet état de chose.

2. L'auteur n'a jamais rencontré de cas où l'hypothèse des parois lisses conduise à des projets incorrects.

3. La valeur limite assignée au rapport (largeur du lit/profondeur) traduit l'absence d'observations sur des rapports plus faibles, mais ne correspond pas à un seuil théoriquement justifié.

4. La nature fait couramment correspondre à des parois cohérentes des pentes dépassant $1 / 1$; les techniciens, eux, adoptent parfois $1 / 2$ ou $1 / 3$. Il est probable que, pour des parois aussi inclinées, l'effort de frottement va en s'accentuant vers la base; l'équation exprimant la constance absolue du frottement tout au long des parois est donc localement en défaut.

5. Dans la pratique courante, l'utilisation dans les formules du débit nominal maximum suffit, puisque le lit cesse d'être actif des que le débit est légèrement inférieur à cette valeur, à moins que le canal ne véhicule des charges tout à fait anormales. Dans cette dernière éventualité, on fera appel à la moyenne probable, à long terme, des débits situés au-dela du point de démarrage supposé des matériaux de fond.

6. Sauf dans le cas des bassins de tête, les fluctuations les plus violentes de la charge solide, aussi complexes qu'elles soient, ne constitueront pas de gêne pour l'ingénieur réglant les canaux. Il y a lieu de s'attendre à de légères fluctuations saisonnières du canal autour de son état de régime moyen; de même on trouvera des séries d'oscillations séculaires. Il suffira de tabler sur une moyenne.

7. Ia distinction physique entre l'absence de tout mouvement de fond et le mouvement le plus faible possible rappelle celle qui existe entre l'écoulement non turbulent et l'écoulement turbulent. Le moindre mouvement du lit donnera lieu à formation de dunes; le coefficient de frottement sera modifié du tout au tout pour s'adapter à ce phénomène et les équations de la théorie du régime deviendront applicables. Elles ne s'appliquent pas s'il y a absence totale de mouvement à tout instant. En cas de doute, on supposera que Je mourement est possible.

8. Il n'y a pas lieu d'admettre que la formation de dunes cesse brusquement au moment précis où la vitesse atteint la valeur critique calculée d'après la profondeur moyenne. La rareté et l'imperfection des observations au voisinage de la vitesse critique nous incite à penser que celle-ci constitue probablement une limite supérieure au-delà de laquelle il n'y aura plus de dunes. La grande dispersion des résultats expérimentaux, jointe aux renseignements que l'on possède sur les rivières et les modèles, nous suggère l'existence d'une zone de transition considérable, le plus souvent en dessous de la zone critiquel et dans laquelle les plus faibles perturbations pourront décider de la présence ou de l'absence de dunes. Heureusement, l'ingénieur qui concoit les canaux ne s'intéressera pas, en général, aux cas où la vitesse est proche de la critique.

9. Parmi les équations du régime, deux pourront être considérées comme des formules de transport solide et servir, par conséquent, dans le cas d'un état provisoirement 
réglé. Mais l'examen de ce cas dépasse l'objet du présent mémoire.

\section{Les trois formules fondamentales de la théorie du régime}

Les trois formules de la théorie du régime ayant une portée pratique revêtent un aspect qui dissimule leur vrai sens; toutefois, pour dresser pratiquement un projet, il est nécessaire de comprendre comment les canaux naturels se règlent eux-mêmes ainsi que les lois auxquelles ils obéissent. Ainsi, nous traiterons dans ce chapitre des trois formules fondamentales dont on a déduit les formules a usage pratique. (Nous avons indiqué succinctement au début les symboles utilisés; si nécessaire, ils seront examinés de plus près dans le texte même du mémoire.)

L'existence de trois équations fondamentales indépendantes se déduit obligatoirement de l'observation de ce qui se passe lorsque l'on creuse, selon un gabarit arbitraire, dans un terrain meuble, un canal destiné à transporter un débit donné avec une concentration donnée en matériaux transportés, quelle qu'en soit la complexité. Sa largeur, sa profondeur et sa pente s'ajusteront de facon que la charge transportée soit véhiculée sans qu'il y ait en moyenne aucun dépôt ou aucune érosion sur le fond ou sur les parois. Cet auto-réglage est en accord avec les lois qui régissent le transport des sédiments. Si donc on les connaît tant soit peu, on pourra exprimer $b, d, \mathrm{~S}$ (largeur, profondeur, pente) en fonction du débit et des paramètres qui définissent la nature du complexe « eau-sédiment » (mixture, suspension, charriage de fond). Il doit donc exister certaines relations entre $b, d, \mathrm{~S}$ et quelques autres grandeurs découlant du régime établi, par exemple la vitesse moyenne $V$, devant permettre de défnir ce complexe eau-sédiment, exactement comme les relations existant entre forces électriques et magnétiques permettent de définir les « quantités »d'électricité et de magnétisme.

Les trois relations dynamiquement satisfaisantes déduites dans le cas de canaux ayant réglé eux-mêmes leurs $b$, $d$ et $\mathrm{S}$ aux valeurs représentant l'équilibre - et considérés dès lor's comme étant «en régime » - peuvent être rationnellement étudiées sous la forme que nous leur donnons ci-après. (Il va sans dire que l'on pourra choisir toute autre trilogie de formules indépendantes résultant d'opérations algébriques.)

1. L Le coefficient de fond $\mathrm{F}_{b}$ peut être défini par :

$$
\mathrm{V}^{2} / d=\mathrm{F}_{b}
$$

relation déduite de ce que $\mathrm{V}^{2} / d$ conserve sa va- leur tout le long d'un système de canaux dans lequel le complexe eau-sédiment reste pratiquement identique.

Le procédé de mesure de $d$ appelle quelques considérations. Un canal rectiligne naturel dont les parois sont en matériaux cohérents et meubles prend une forme pouvant être considérée comme trapézöidale; son fond est constitué par des dunes en mouvement. Pour effectuer la mesure exacte de la profondeur moyenne $d$ d'un canal existant, il faudrait observer des profils en travers échelonnés sur plusieurs longueurs de dune, a des intervalles convenablement choisis pour qu'il n'y ait pas prépondérance de crêtes ou de creux; ou bien on observera a plusieurs reprises des profils en un seul point. C'est le premier des deux procédés qui est ordinairement utilisé au laboratoire; mais, sur place, l'ingénieur en mission de jaugeage penche vers le second, peut-être inconsciemment, en relevant quelques profils en un même point lors de chaque relevé périodique et en constatant qu'il n'y a pas à modifier sensiblement le niveau moyen du lit pour passer d'un enregistrement à un autre. En creusant le canal, on lui assigne la profondeur que donne le calcul de $d$; si les prévisions du calcul étaient exactes, la profondeur effective qui s'établit d'elle-même ne devrait pas s'en écarter suffisamment pour être prise en considération.

La vitesse moyenne $V$ est celle que l'on déduit du débit $Q$ et de la section trapézoidale dont la profondeur entre le niveau de la surface libre et le fond moyen est $d$.

Souvent, pour les besoins de la pratique, i] suffira d'une seule observation soigneuse de section pour connaître $d$.

2. - Le coefficient de paroi $\mathrm{F}_{s}$ peut être défini par :

$$
\mathrm{V}^{3} / b=\mathrm{F}_{\mathrm{s}}
$$

$b$ pouvant être pris, par souci de commodité, comme la largeur qui, après multiplication par $d$, donne l'aire de la section trapézoïdare moyenne; pratiquement, on pourra le considérer comme désignant la largeur à mi-profondeur. La formule ci-dessus résulte de la transformation d'autres formules, non d'observations directes qui ne s'avéreráient guère commodes du fait que la nature des parois varie notablement d'un point à l'autre.

Si l'on multiplie $F_{s}$ par le carré de la masse spécifique et par la viscosité cinématique, le produil obtenu aura les dimensions du carré d'une tension: cette tension semble donner la mesure de l'effort tangentiel appliqué aux parois, si elles sont « lisses », hydrauliquement parlant. Il semble cependant assez bizarre que les parois bien entretenues soient « lisses », alors que le matériau dont elles sont faites peut 
être considéré au laboratoire comme «rugueux». Mais le paradoxe disparait si l'on se rappelle que le caractère lisse ne dépend pas de la hauteur des rugosités, mais de leur hauteur relative, hauteur relative bien moins grande sur le terrain qu'au laboratoire; on trouve même de grands tunnels en béton fonctionnant en régime quasi lisse, alors que des tuyaux en béton de dimensions usuelles se situent dans le domaine rugueux.

3. - La formule donnant le débit représente une généralisation de celle de Blasius appliquée aux conduites lisses; elle s'écrit :

$$
\mathrm{V}^{2} / g d \mathrm{~S}=3,63(1+a \mathrm{C}) \cdot(\mathrm{V} b / v)^{0,25}
$$

$a \mathrm{C}$ pouvant être négligé dans la majorité des canaux artificiels de la pratique. Qu'une formule du type «lisse » s'avère correcte est évident, après coup. Le terme malencontreux de «lisse», lorsqu'il s'agit de l'hydraulique des parois rigides, signifie simplement qu'au voisinage de la paroi le fluide présente un comportement mécanique tel que la résistance à l'écoulement peut être calculée en fonction de ses caractéristiques propres - la viscosité, en l'espèce - alors que la paroi ne joue qu'un rôle de support. Dans un canal à l'état de régime, le fond et la zone avoisinante doivent être distingués du complexe eau-sédiment.

Le terme $(1+a \mathrm{C})$ a été ajouté récemment par l'auteur pour des raisons données par ailleurs [12]. La formule primitive, mais sans ce terme, a été trouvée par C. KING 113$\rceil$ d'après des observations sur un canal d'irrigalion pour lequel $C$ était trop faible pour être pris en considération. La valeur de « $a$ » est voisine de $1 / 400$ pour un sable homogène, comme celui qui était employé au cours des expériences classiques réalisées par Grubert sur des canaux de laboratoire [4]. Faute de données, les auteurs ne peuvent proposer de valeur précise pour le cas des sables et graviers naturels; ils recommandent d'essayer avec 1/233. C'est, mesuré en millièmes de pourcent (en poids), le rapport entre le poids (dans l'air) du matériau de fond passant par unité de temps et le poids de l'eau passant par unité de temps. On pourrait le nommer concentration, mais ce terme recèle l'idéc d'une distribution uniforme; aussi Ini a-t-on affecté le terme spécial de « charge ».

\section{Les trois formules fondamentales de la théorie du régime entrant dans les calculs}

Le problème-type du calcul pratique d'un canal peut être énoncé de la façon suivante:

«Etant donnés : le débit, la nature du complexe eau-sédiment exprimée au moyen rle la viscosité cinématique et du coefficient de fond, ainsi que la sensibilité des parois à l'érosion qu'exprime le coefficient de paroi, trouver la largeur, la profondeur et la pente pour lesquels le canal peut fonctionner en état de régime. 》

Pour résoudre la question sans calculs algébriques superflus, on transformera les équations (1) à (3) de facon qu'elles domnent $b, d$ et $\mathrm{S}$ directement en fonction de $Q, F_{b}$ et $F_{s}$. Le plus simple est d'écrire (1) et (2) sous la forme:

$$
\text { d'oì : } \begin{gathered}
\left(\mathrm{V}^{2} / d\right) \cdot\left(\mathrm{V}^{3} / b\right) \cdot(\mathrm{V} / \mathrm{V})=\mathrm{V}^{6} / \mathrm{Q}=\mathrm{F}_{b} \cdot \mathrm{F}_{s} \\
\mathrm{~V}=\left(\mathrm{F}_{b} \cdot \mathrm{F}_{s} \cdot \mathrm{Q}^{)^{16}}\right.
\end{gathered}
$$

Introduisant alors la valeur de $\mathrm{V}$ dans les trois équations fondamentales, nous obtiendrons :

$$
\begin{aligned}
& b==\left(\mathrm{F}_{b} \mathrm{Q} / \mathrm{F}_{s}\right)^{1 / 2} \\
& d=\left(\mathrm{F}_{s} \mathrm{Q} / \mathrm{F}_{b^{2}}\right)^{1 / 3} \\
& \mathrm{~S}=\frac{\mathrm{F}_{b}^{5 / 6} \mathrm{~F}_{s}^{1 / 12} v^{1 / 4}}{3,63(1+a \mathrm{C}) g \mathrm{Q}^{1 / 6}}
\end{aligned}
$$

où « $a$ »est très peu connu, mais peut être pris égal à $1 / 233$; le terme $(1+a \mathrm{C})$ dans son ensemble ne semble pas devoir s'écarter beaucoup de l'unité, sauf dans les canaux véhiculant des charges tout à fait anormales.

Les équations (A), (B) et (C) constituent les formules fondamentales pratiques de la théorie du régime. On trouvera, par ailleurs, la facon d'evaluer $F_{b}$ et $F_{s}[1],[2],[10]$.

\section{Comparaison entre les résultats de la théorie du régime et ceux obtenus en laboratoire}

Le tableau $n^{\circ}$ I compare les gammes respectives de certains paramètres fondamentaux découlant d'une part de la théorie du régime et, d'autre part, des résultats expérimentaux de laboratoire; les uns sous la rubrique « Canaux», les autres sous les titres « Gilbert», « MeyerPeter $»$ et «Einstein $»$; les renseignements concernant les essais de laboratoire avec des sables naturels de rivière ne sont pas portés, mais n'apporteraient guère d'éléments nouveaux à cette comparaison, sauf en ce qui concerne la granuloméfrie. Il convient de noter les remarques suivantes :

1. - Les deux gammes respectives de données se complètent largement. Ainsi, pour le nombre de Reynolds relatif à la largeur, si significatif, les canaux s'étendent sur deux bandes successives d'exposant, alors que les essais de laboratoire couvrent une bande voisine. Les canaux couvrent une gamme très large de débit allant de 0,03 à $300 \mathrm{~m}^{3} / \mathrm{s}$, alors que les essais de laboratoire s'étendent de 0,003 à $0,03 \mathrm{~m}^{3} / \mathrm{s}$. Les ca- 
TABLEAU I. -- LES GAMMES D'OBSERVATIONS

\begin{tabular}{|c|c|c|c|c|}
\hline & Canaux Naturels & GILBERT & Meyer-Peter & EINSTEN \\
\hline 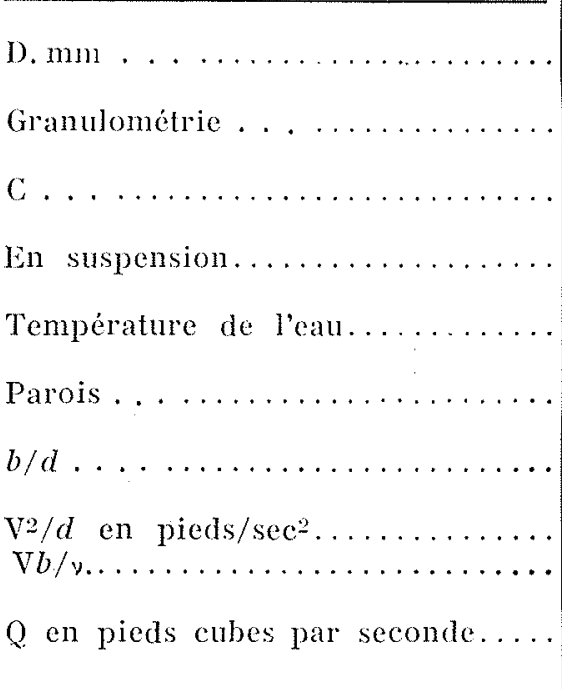 & $\begin{array}{l}0,10-0,60 \\
\text { Probabilité log. } \\
0 \text { à } 2 \text { ou } 3 \\
0 \text { à } 1 \% \\
10-30^{\circ} \mathrm{C} \\
\text { Argile, lisse } \\
4 \text { à } 30 \\
0,5 \text { à } 1,5 \\
10^{6} \text { à } 10^{\circ} \\
1 \text { à } 10.000\end{array}$ & $\begin{array}{l}0,3-7,0 \\
\text { uniforme } \\
0 \text { à } 3.000 \\
0 \\
\text { Prob. } 13^{\circ} \mathrm{C} \\
\text { Bois ou verre, lisse } \\
1 \text { à } 25 \\
1 \text { a } 150 \\
10^{5} \text { à } 10^{6} \\
0,1 \text { a } 9,0\end{array}$ & 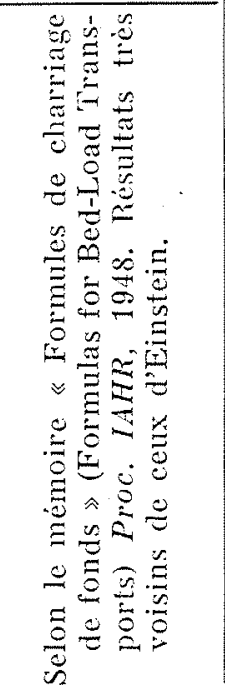 & 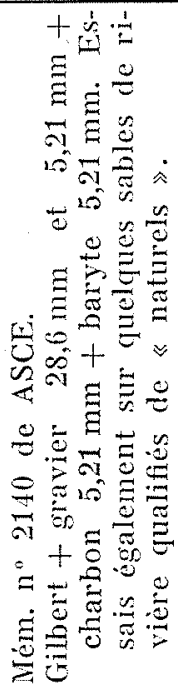 \\
\hline Régime. & Dunes & \multicolumn{3}{|c|}{ Dunes, fond lisse et antidunes } \\
\hline
\end{tabular}

naux ne comportent que des dunes, alors que les essais sur modèle révèlent des écoulements à fond lisse et à antidunes. Pour les canaux, les charges atteintes par le charriage de fond sont insignifiantes et échappent même aux mesures, tandis que les canaux de laboratoire véhiculent jusqu'à $3 \%$ en poids, ce qui ne se présente dans la nature que dans des cas très rares. Le coefficient de fond des canaux (exactement proportionnel au nombre de Froude relatif à la profondeur) va de 0,5 à 1,5 ; en laboratoire il varie de 1 à 150 .

2. - Le coefficient de parois, correspondant au cas "lisse », est le même dans les deux cas; sinon, toute comparaison des données serait aléatoire, ou même impossible.

3. - On constate une lacune dans les deux cas à propos du calibre des grains. Aux yeux du spécialiste de l'hydraulique fluviale projetant des ouvrages dans des lits de gravier ou de cailloux, c'est-à-dire ayant affaire à des matériaux allant par exemple de 25 à $300 \mathrm{~mm}$, le domaine des canaux entre 0,1 et $0,60 \mathrm{~mm}$ et celui des essais en laboratoire, de 0,3 à $7,0 \mathrm{~mm}$ (avec une singularité à $28,6 \mathrm{~mm}$, singularité pour laquelle les auteurs n'ont pas réussi à obtenir des données hydrauliques originales) sont comparables et l'un et l'autre insuffisants.

4. - Les résultats obtenus au laboratoire avec des matériaux uniformes peuvent conduire à des relations fonctionnelles correctes, mais ne donneront pas des valeurs de coefficients applicables aux sables de rivière naturels.

Et l'on est conduit ainsi à des réflexions intéressantes. Tout d'abord, il n'y a rien d'étonnant à ce que les diverses formules découlant de ces deux jeux de résultats ne concordent pas parfaitement quand on extrapole les unes dans le domaine des autres. Ensuite il est de première importance de chercher à raccorder les deux domaines, soit par des formules nouvelles, soit par des formules anciennes modifiées sans pour autant les vider de leur signification dynamique. Enfin, lors même que l'on arriverait à couvrir avec un seul groupe de formules les deux domaines, les lacunes concernant le calibre des grains laissent subsister un doute considérable sur leur validité lorsqu'il s'agit de canaux véhiculant des graviers et des cailloux; il y a donc lieu de poursuivre l'étude. Et ce qui vient d'être dit porte l'atlention sur une particularité de la formule Meyer-Peter; celle-ci prétend évaluer la quantité de matériaux transportés sur une largeur-unité d'un canal dont le débit, la pente et la granulométrie seraient donnés; et ceci s'appliquerait aussi bien à un matériau de 6 pouces $(15 \mathrm{~cm})$ dans une rivière profonde de 30 pieds $(9 \mathrm{~m})$ qu'au même matériau dans un canal profond de 1 pied $(0,3 \mathrm{~m})$; il n'y a là, semble-t-il, rien d'en lui-même absurde, mais cette assertion remarquable mérite d'être contrôlée sur un matériau plus gros que 7,0 mm. 


\section{Analyse préliminaire, par la théorie du} régime, des observations de Gilbert (12)

Vis-à-vis de la théorie du régime, les observations de Grubert [4] constituent quelque mille « observations de canaux en état de régime » avec parois lisses comme d'ordinaire. Elles bénéficiaient, cependant, de la possibilité de mesurer la grosseur des matériaux de fond $\mathrm{D}$, comme aussi les charges transportées $\mathrm{C}$ ( $\mathrm{C}$ étant le rapport entre le poids des matériaux de fond et le poids de l'eau passant dans l'unité de temps, exprimé en millièmes de pourcent, ou en centmillièmes); il n'y avait pas lieu de faire intervenir ici le «coefficient de paroi » (équation 2) puisque ces parois n'étaient pas auto-réglables. L'étude préliminaire consistait done à reconnaître :

1. --- Si le coefficient de fond, $F_{b}=V^{2} / d$ peut être représenté comme une fonction de $\mathrm{C}$ el de $D$, sans préjuger pour autant de l'influence possible de $b / d, \mathrm{D} / d$ et de la viscosité.

2. - Si, dans la formule de pente de la théorie du régime, établie d'après les canaux naturels où $\mathrm{C}$ est très petit, c'est-à-dire :

$$
\mathrm{V}^{2} / g d \mathrm{~S}=3,63(\mathrm{~V} b / v)^{0.25}
$$

la constante 3,63 doit être modifice en fonction de $C$ sans préjuger pour autant de linfluence possible de $b / d$ et $\mathrm{D} / d$.

\section{Examen du POINT 1}

Il était possible, d'abord, de vérifier rapidement la possibilité d'une relation concernant le coefficient de fond, en groupant les résultats correspondant à un calibre donné de matériau, et en dressant un tableau destiné à mettre en évidence :

a) le passage du régime des dunes au régime à fond lisse,

b) le passage du régime à fond lisse au régime des antidunes.

Il semblait normal que l'on assiste à une modification du régime pour un nombre de Froude bien défini ou, ce qui revient au même, pour un certain coefficient de fond. Ceci vérifié, on devait s'attendre aussi à voir cette modification intervenir pour une certaine charge du charriage de fond, quelles que soient les valeurs globales du débit et la charge globale. Nous avons donc porté $V$ en fonction de $d$, et la charge en fonction du débit pour chaque point de transition, ou chaque «point critique», selon la terminologie de Grlbert. Le premier diagramme fait apparaitre une relation parabolique, dans un cas comme dans l'autre; le second révèle une relation linéaire dans les deux cas aussi. Cela confirmait la propriété du coefficient de fond d'être un bon critère de changement de phase, même au-dela des conditions correspondant aux canaux naturels, cela confrrmait également l'importance de la variable «charge» dans la relation exprimant ce coefficient de fond.

Encouragés par ces résultats, nous avons abordé un deuxième stade et porté, sur du papier quadrillé ordinaire, en fonction de la charge, les coefficients de fond correspondant à tous les matériaux de Grmbert depuis A jusqu'à $\mathrm{H}$ (di-

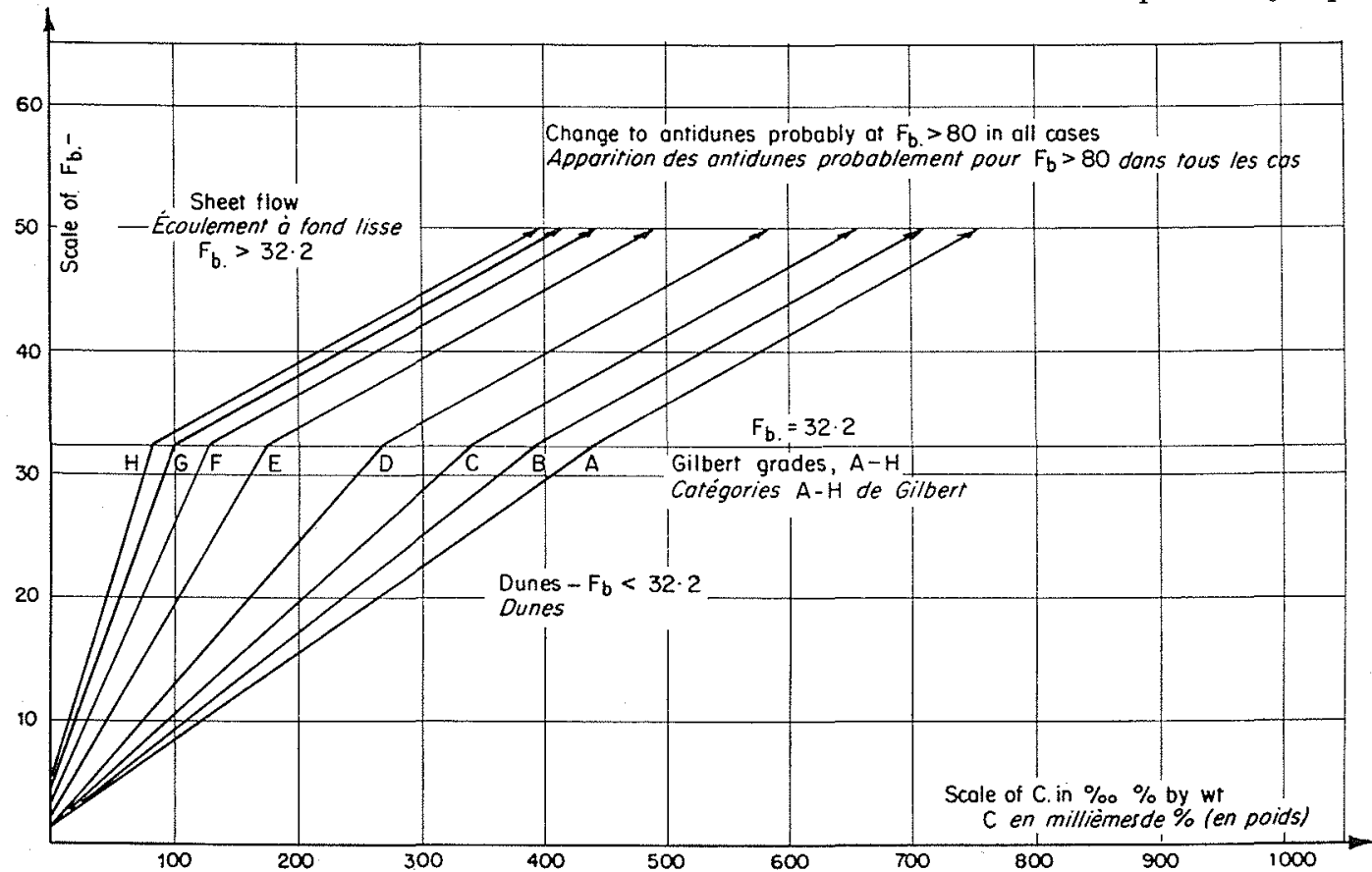

Fig. 1. - Etude préalable. Coefficient de fond en fonction de la charge. Sables uniformes de Gilbert. Gilbert uniform sands. 
mensions 0,30 à $7,0 \mathrm{~mm}$ ) en affectant des indices particuliers à chacun des matériaux. La figure 1 montre le réseau des courbes finalement déduites de ce pointage. Elles représentent une des façons d'utiliser les résultats d'observation; elles ont aussi le mérite de constituer une étape vers une analyse plus approfondie et de mettre en évidence l'insuffisance de certains résultats existants pour vérifier une hypothèse quelconque. Afin d'éviter les fausses interprétations, nous donnons ci-après, avec quelques détails, l'histoire de l'évolution de ces courbes.

A mesure que nous avancions dans le tracé des graphiques, la nature des valeurs données s'éclaircissait. La dispersion était, dans l'ensemble, aussi grande que la laissaient prévoir les données que nous possédions relativement aux canaux naturels. Dans la zone de dunes, elle était plus forte que dans celle du fond lisse; dans la zone des antidunes, elle était si décevante gu'il a fallu laisser tomber les points correspondants. Pour certains matériaux, les données étaient très clairsemées dans la zone des dunes; et, pour tous les matériaux, ces données étaient impropres, dans la région des faibles charges, à indiquer une valeur probable de $\mathbf{F}_{b 0}$, c'est-à-dire de $F_{b}$ quand la charge tend vers zéro. C'était bien malheureux, car les réalisateurs de canaux s'intéressent surtout à $F_{b 0}$ et les théoriciens préfèrent partir d'une courbe aussi bien définie que possible. Toutefois, l'homme de la pratique n'ignore pas que, pour les sables et des charges insignifiantes, on peut adopter une valeur empirique approchée de $F_{b_{0}}$ :

$$
\mathrm{F}_{b 0}=1,9 . \mathrm{D}_{m}^{0,5}
$$

$D_{m}$ indiquant les dimensions moyennes en $\mathrm{mm}$ du matériau de fond et $F_{b n}$ étant exprimé, comme de coutume, en pieds/sec 2 . Il hésiterait, avant de l'employer pour des matériaux de $7 \mathrm{~mm}$ par exemple, mais un simple coup d'œil sur la figure 1 lui montrerait qu'une erreur de $50 \%$ conduirait à un résultat à peine différent des conclusions de la relation fonctionnelle donnant la charge. Nous avons donc utilisé cette formule pour fixer la position probable de l'origine des courbes. [Le fait qu'on arrive à la figure 1 si convaincante ne change en rien le caractère grossier de l'équation (4).]

Tous les points ayant été portés sur le diagramme et ses limites inférieures ayant été fixées d’après la formule, le tracé général des courbes apparaît peu à peu tel que sur la figure 1 , avec des lignes parallèles dans la région supercritique où $\mathrm{F}_{b}$ est supérieur à $g=32,2$ pieds/ $\mathrm{sec}^{2}$ et d'autres plutôt mal définies (pour certains matériaux surtout) dans les zones subcritiques. Le parallélisme supercritique était suffisamment marqué pour donner l'impression qu'il n'était pas dû au hasard. Dans ce cas, le subcritique doit obéir à une autre loi, aucun effort d'imagination ne permettant d'admetre que les lignes parallèles de la partie supérieure puissent conduire à des valeurs de $\mathrm{F}_{b 0}$ voisines de la réalité. L'hydraulique classique donne à penser que les modifications de régime s'accompagnent d'une zone de transition, mais étant donné la dispersion des données, il semblait préférable de mettre en évidence une variation discontinue. On a situé cette discontinuité du point de vitesse critique, c'est-à-dire quand $\mathrm{F}_{b}=g$, parce que c'est là une caractéristique hydraulique bien connue et aussi parce que les observations semblaient indiquer que le passage du régime des dunes au fond lisse avait effectivement lieu au voisinage de cette vitesse. Ce choix a été fait, bien que la brève vérification mentionnée au début du chapitre ềt montré que ce passage correspondait à une valeur de $\mathrm{F}_{7}$, très supérieure; à ce sujet, Grcbistit dit que " la position des points critiques a été définie par M. Murphy à une époque où les détails expérimentaux étaient encore présents à la mémoire $»$. Ce conflit entre la mémoire el les valeurs relevées a pu trouver son explication à l'époque et provenir de ce que M. Murphy se réservait une marge de sécurité dans le cadre de certaines règles rationnelles; aujourd'hui, nous ne pouvons que l'enregistrer et en tenir comple dans le cas de résultats aberrants. A ce stade, la figure 1 comprenait les points de départ inférieurs, les courbes mal définies dans la zone suberitique, les discontinuités correspondant à la période critique et les droites parallèles de la zone supercritique.

I'étape suivante consistait à examiner si l'on pouvait remanier les courbes jusqu’à les réduire à une seule courbe discontinue, par l'introduction de variable sans dimensions pouvant conduire à l'équation d'une famille déterminée, d'après laquelle on reconstruirait la figure 1 avec, cette fois-ci, des courbes bien définies. Nous avons admis simplement que, dans la phase subcritique du moins, on a :

$$
\mathrm{F}_{b}=\mathrm{F}_{b 0}[1+f n(\mathrm{C})]
$$

$f_{n}(\mathrm{C})$ étant destiné à tendre vers zéro en même temps que $\mathrm{C}$ et à traduire le fait physique que $F_{b 0}$ n'est pas nul lorsque $C$ s'annule et est approximativement donné par l'équation (4). Il fallait donc, de toute évidence, tracer :

$$
\left(\mathrm{F}-\mathrm{F}_{b 0}\right) / \mathrm{F}_{b 0},
$$

(rapport qui pourrait être désigné par « écart relatif du coefficient de fond $\$$ ), en fonction de C. C'est ce que nous avons fait, pour le régime en dunes seulement, sur papier logarithmique double; et une relation tout à fait définie est apparue, mais la dispersion de certains points était trop grande pour décider nettement si le tracé le plus exact était une droite ou une légère courbe. (Fort heureusement pour l'étape à 
venir, il n'y avait guère de doute quant aux charges correspondant à la vitesse critique qui servaient à la vérification du paxallélisme en zone supercritique.) Il n'y avait pas de point où la charge fût inférieure à 8 ou supérieure à 500 ; les plus nombreux se trouvaient aux charges supérieures à environ 80 . On décida donc de faire passer par les points une courbe :

$$
\mathbf{F}_{b}=1,9 \mathrm{D}_{m}^{0,5}(1+0,068 \mathrm{C})
$$

bien qu'une relation non linéaire en $C^{5 / 6}$ eût $\mathrm{pu}$ tout aussi bien convenir. Les courbes subcritiques que l'on voit sur la figure 1 ont été tracées à l'aide de cette équation et arrêtées à :

$$
\mathrm{F}_{b}=g=32,2 \text { pieds } / \mathrm{sec}^{2}
$$

La dernière étape comprenait le tracé de $\mathrm{F}_{b}-g$ en fonction de $\mathrm{C}-\mathrm{C}_{c}$, pour les points supercritiques seuls, $C_{\theta}$ étant la charge telle que déduite de l'équation (6) en posant $\mathrm{F}_{b}=g$. Pour ce contrôle du parallélisme des lignes supercritiques, nous avons utilisé du papier quadrillé ordinaire, gradué en $\mathrm{C}-\mathrm{C}_{c}$ entre -80 environ et +1.700 , les points les plus nombreux étant en dessous de 800 . La courbe obtenue s'avéra bien une droite passant par l'origine et ayant pour équation :

$$
\mathrm{F}_{b}-g=0,056\left(\mathrm{C}-\mathrm{C}_{c}\right)
$$

Sur la figure 1, les lignes supercritiques ont été tracées d'après cette équation.

Les conclusions finales à tirer étaient que le coefficient de fond constitue un critère valable jusqu'aux charges donnant naissance aux antidunes (ces charges n'étant pas nécessairement comprises), et que la figure 1 peut passer pour une représentation convenable de la relation fonctionnelle liant le coefficient de fond au calibre des matériaux et à la charge, tanl qu'on ne possédera pas de données meilleures.

\section{EXAMEN DU POINT 2}

Pour vérifier l'exactitude de l'équation (3a), nous avons calculé les valeurs de :

$$
\mathrm{K}=\mathrm{V}^{2} / g d \mathrm{~S} \text { divisé } \operatorname{par}(\mathrm{V} b / \nu)^{0,23}
$$

donnant 3,63 pour les canaux naturels sur lesquels était basée la théorie du régime et nous les avons portées ensuite en fonction de $\mathrm{C}$ sur papier quadrillé ordinaire. Nous avons ainsi obtenu, dans le domaine subcritique, une droite indisculable passant par la valeur 3,63 pour $C$ égal à zéro. Les points au-delà du critique étaient éparpillés sans ordre, mais semblaient quand même osciller autour d'une valeur constante pour certains matériaux et tomber nettement pour certains autres. De même que dans l'examen du point 1 , les points antidunes étaient omis.

L'équation de la courbe dans la zone subcritique était :

$$
\mathrm{V}^{2} / g d \mathrm{~S}=3,63(1+\mathrm{C} / 400)(\mathrm{V} b / \mathrm{v})^{0,25}
$$

formule sur laquelle nous avions anticipé en écrivant l'équation (3) au début de cet article. Le diagramme sur papier quadrillé ordinaire se présentant sous un aspect peu maniable, nous avons été obligés de le reporter sur papier loga-

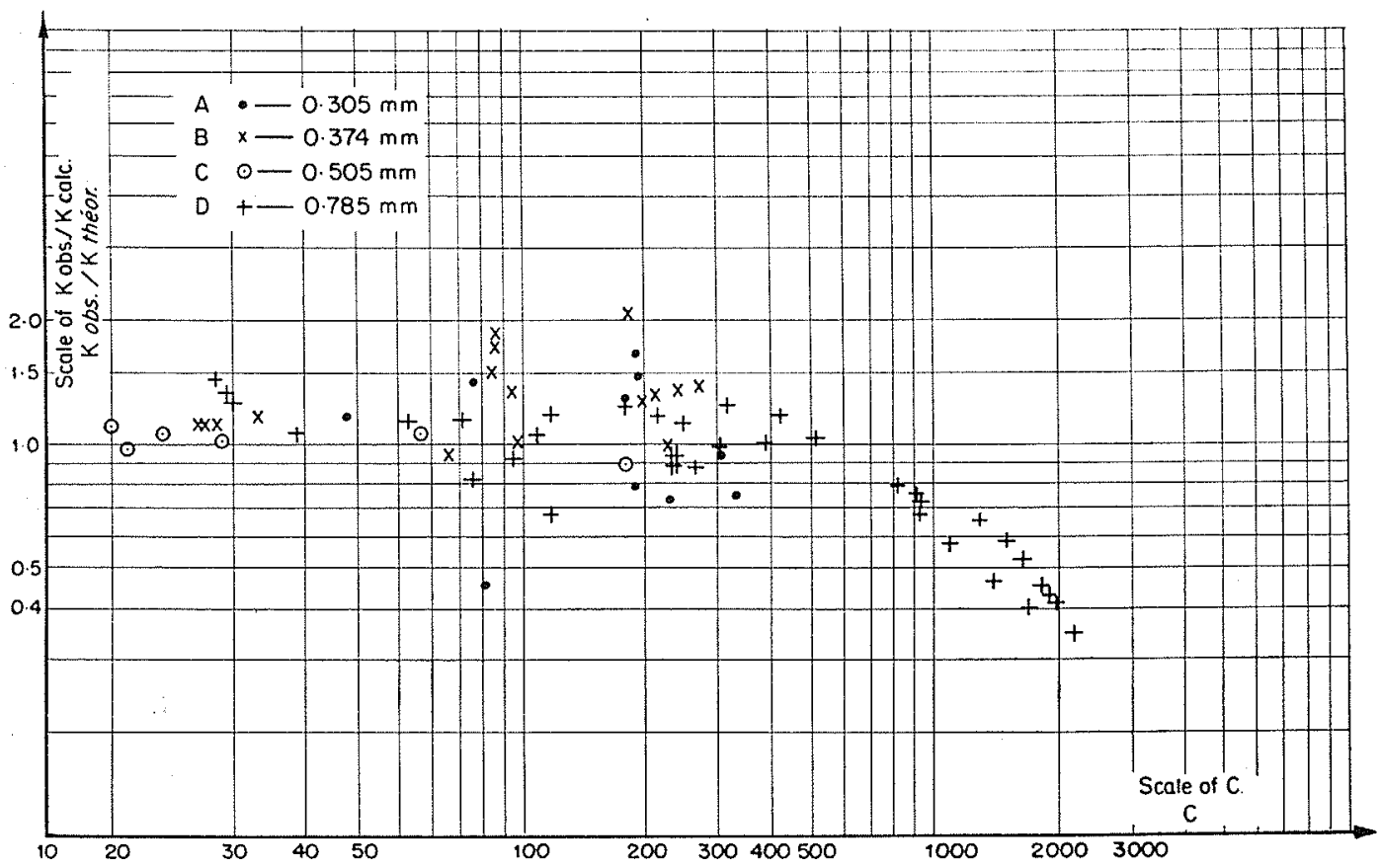

FIG. 2. - Etude préalable. Observations de K pour le régime en dunes. Sables uniformes de Gilbert.
Preliminary Analysis. Test of $K$ for dunes. Gilbert uniform sands. 
rithmique double, pour la publication (fig. 2); cela permet de juger si $\mathrm{V}^{2} / g d \mathrm{~S}$ divisé par $(\mathrm{V} b / v)^{0,25}$ fournit des points réellement dispersés autour de $1+(\mathrm{C} / 400)$. La figure a été tracée dans la zone supercritique pour le matériau D, lequel donne les valeurs les plus compatibles montrant comment l'équation (3 $a$ ) devient brusquement inapplicable quand s'établissent les conditions critiques de l'écoulement.

On arriverait donc finalement aux conclusions suivantes :

a) La constante 3,63 , qui entre dans la formule de la théorie du régime, est en réalité une fonction de la charge et, dans le cas de la zone subcritique et d'un sédiment unilorme se rangeant dans les gammes définies plus haut, elle peut être modifiće en :

devenant :

$$
3,63[1+(\mathrm{C} / 400)]
$$

$$
3,63(1+a C)
$$

s'il s'agit d'un sédiment quelconque conservant son caractère pendant la durée de l'écoulement, le facteur a devant ètre déterminé par l'observation.

b) Les résultats connus ne peuvent conduire à une relation pour l'écoulement supercritique (probablement à cause de l'imprécision des mesures de pente au laboratoire), mais ils montrent clairement que la relation trouvée pour le cas subcritique est inapplicable au cas supercritique.
Le fait que, par simple adjonction d'un multiplicateur, la formule de pente de la théorie du régime soit rendue valable pour la gamme des fortes charges correspondant aux essais de laboratoire, confirmait si fermement son exactitude formelle au point de vue dynamique que l'établissement de la figure 3 visait en partie les besoins de la pratique, en partie une demonstration du processus de la généralisation. La théorie du régime sous sa forme primitive domnait la droite de charge nulle (zéro) pour les nombres de Reynolds compris entre $10^{6}$ et $10^{8}$. Les résultats de laboratoire donnaient les lignes d'égale charge dans la gamme comprise entre $10^{*}$ et $10^{6}$. Les droites d'égale charge ont été prolongées jusque dans le domaine couvert par la théorie du régime, en s'appuyant sur le fait que la formule établie n'avait rien perdu de son caractère original ou de ses significations dynamiques possibles. Le lecteur ne devra pas étendre cette figure au cas de la pratique sans certaines précautions, car les droites situées audessus de la droite zéno seraient autrement espacées dans le cas des sables naturels [10]. Si l'on s'en tient aux résultats connus à ce jour, la position de la droite zéno ne change pas beaucoup quand on passe d'un sable uniforme à un sable naturel.

\section{Seconde étude [11]}

पा

L'analyse préliminaire s'était limitée aux observations de GrLbert concernant des sables et

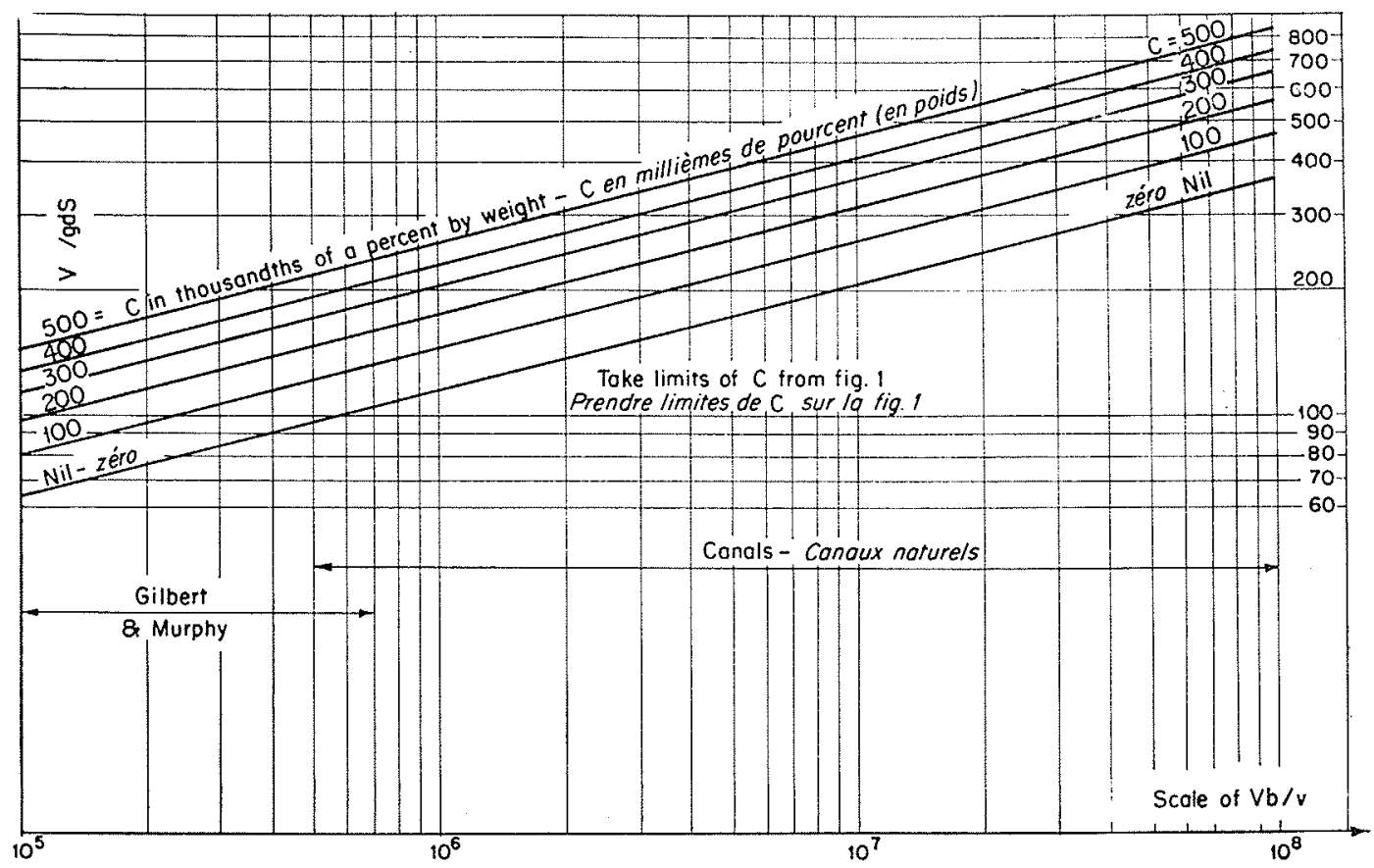

Fic. 3. - Extension de la formule de pente des canaux naturels en régime, de façon à l'adapter aux sables uniformes de Gilbert (dunes seulement).
Canal regine slope formula extended to suit Gilbert uniform sands (dunes only). 
des graviers fins rendus « uniformes » artificiellement. Elle méconnaissait les points antidunes, plusieurs groupes de points « fond lisse » qui ne semblaient pas apporter une contribution utile aux diagrammes (elle économisait du travail en les considérant comme dus au hasard), ainsi que quelques points dont les observations laissaient en suspens la question de savoir s'ils appartenaient à l'état dunes ou fond lisse. On se décida donc à reprendre l'analyse de l'ensemble des résultats de GiLnenT à tête reposée et à rechercher d'autres résultats notamment dans le domaine des matériaux naturels de rivière.

En cherchant à trouver des observations non gilbertiennes, nous avons constaté combien peu de besogne effective a été faite depuis l'année 1914 qui est celle de ses publications, ainsi que la supériorité de ses méthodes, de sa technique et de sa présentation. La documentation ultérieure où nous avons cherché à puiser des informations souffre toujours d'un ou de quelques-uns des défauts suivants :

1. Canaux expérimentaux trop courts pour que les profondeurs aient acquis l'état d'uniformité voulue;

2. Canaux trop courts pour que les pentes puissent être considérées comme caractéristiques vis-à-vis des faibles parcours d'écoulement uniforme;

3. Impossibilité d'établissement d'un régime permanent;

4. Utilisation de matériaux artificiels, de telle sorte que l'on se trouve simultanément en présence de suspensions et de charriages de fond, les mesures étant faites comme s'il n'y avait que l'un ou l'autre;

5. Confusion entre les conditions de démarrage du mouvement de fond et les conditions postérieures au démarrage;

6. Gamme d'essais très resserrée;

7. Gamme plus large mais forte dispersion non compensée par le nombre d'observations;

8. Résultats originaux inacessibles.

Ainsi donc, pour des matériaux uniformes, on ne disposait que des valeurs de Ginbert, ou elles étaient seules acceptables; pour les matériaux de rivière naturels, on n'avait que les résultats donnés par G. L. Straub [16] el le Corps du Génie des Etats-Unis [17].

1. Facteur de rond, d'apres Gilbert. - Pour ne pas se montrer trop enclin à admettre une discontinuité de la loi aux vitesses critiques, comme sur la figure 1, nous' avons décidé d'établir des diagrammes donnant l'écart relatif des coefficients de fond $\left(\mathrm{F}_{b}-\mathrm{F}_{b 0}\right) / \mathrm{F}_{b 0}$ en fonction de la charge pour chacun des points de GILBERT. La fioure 4 en est un exemple, dans le cas des matériaux du type D. La figure 5 montre les courbes déduites de tous les diagrammes et tenant compte, en plus, des résultats concernant les matériaux naturels.

Il n'est pas surprenant qu'à la figure 1 il y ait une discontinuité et qu'à la figure 5 il n'y en ait point. Tout d'abord, lorsqu'il s'agit de faire passer une courbe par des valeurs très dis-

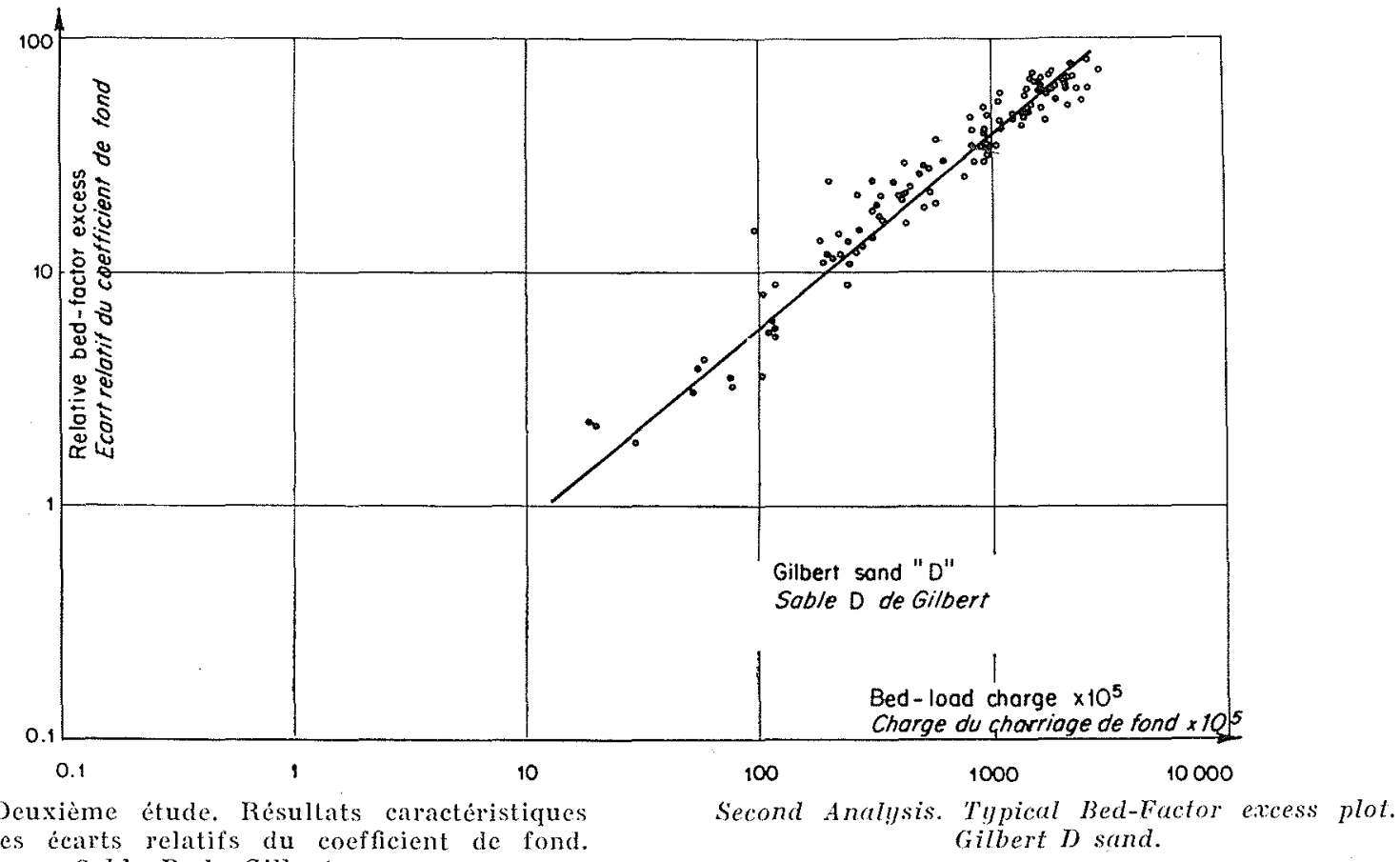

Fic. 4. - Deuxième étude. Résultats caractéristiques
concermant les écarts relatifs du coeffeient de fond. Sable D de Gilbert. 


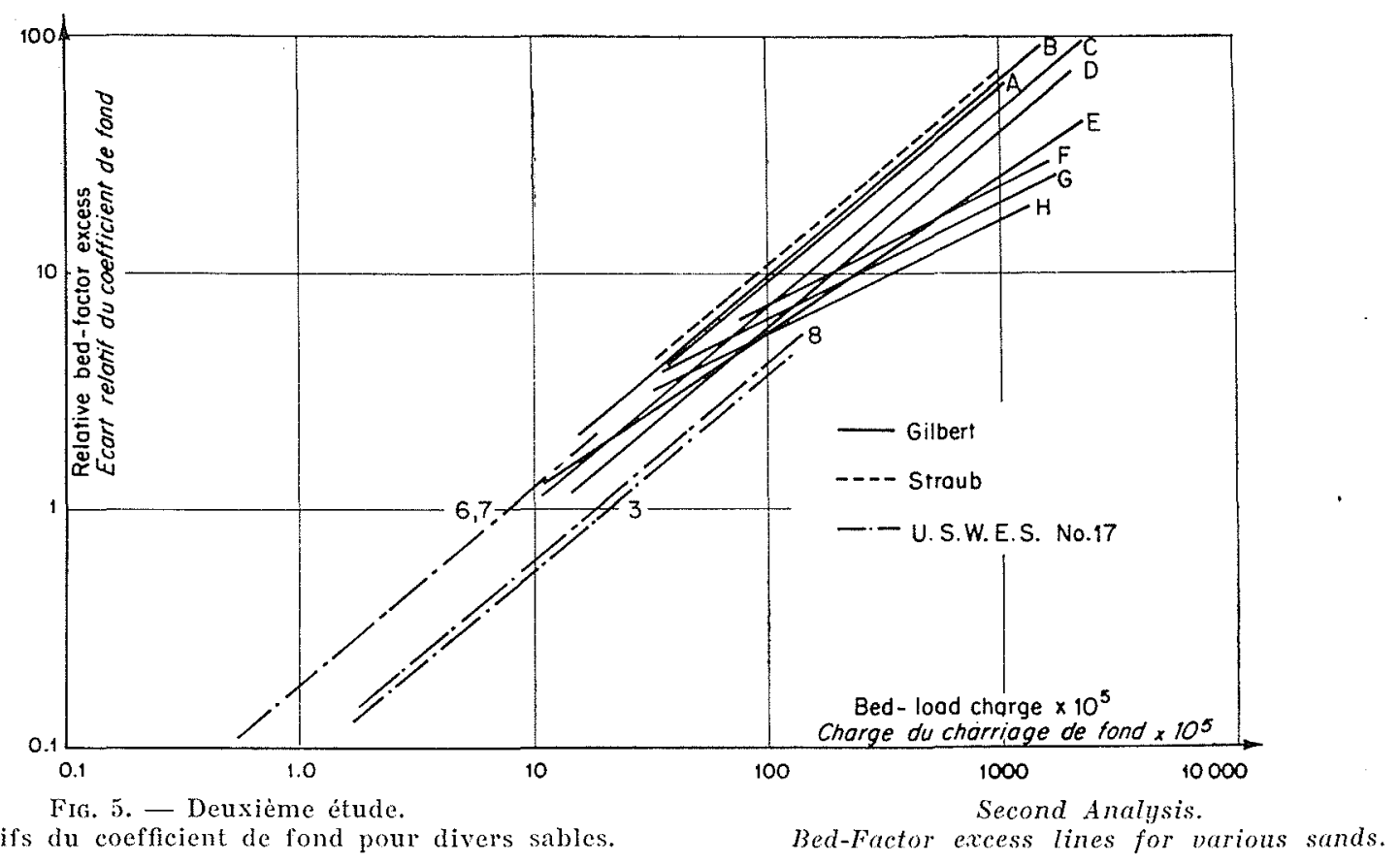

Ecarts relatifs du coefficient de fond pour divers sables.

persées, même avec une seule variable indépendante, on trouvera plusieurs courbes inspirées "d'hypothèses physiques différentes, et toutes situées à l'intérieur de la zone délimitée par les considérations de la statistique. Ensuite, les différents procédés mathématiques pour trouver les équations de ces courbes sont comme autant d'instruments d'optique (lentilles, miroirs déformants, etc.) que nous employons pour: observer les objets : ils lissent facilement les courbes et masquent les discontinuités. (En particulier, si l'on suppose que les droites subcritiques de la figure 1 étaient remplacées par des paraboles de puissance $5 / 6$ considérées comme tout aussi bonnes (voir équation 6 ci-après), leur aspect ne changerait pas beaucoup. Transposées dans le système de coordonnées de la figure 5, ces nouvelles courbes deviendraient des droites de pente $5 / 6$ tout à fait semblables aux droites $A$ à $\mathrm{D}$, peut-être un peu décalées, sans changement de pente; elles ne seraient pas conformes aux lignes $\mathrm{E}$ à $\mathrm{H}$, ce que l'on décèlerait tres difficilement du fait que ces courbes ne s'appuient pratiquement sur aucun point dans la zone subcritique. Il résulte de ce qui précède qu'il y a pénurie de points pour les matériaux fins dans la zone des antidunes, que les matériaux grossiers en manquent considérablement dans la zone des dunes, et que les uns et les autres en manquent dans la zone des faibles charges où il $\mathrm{y}$ en aurait tant besoin pour bien amorcer les courbes.

Au cours de l'analyse en vue d'établir l'allure exacte des courbes de la figure 1, nous avons été amenés à tracer une figure 5 pour la zone subcritique, mais en faisant passer une seule courbe par les différents points, au lieu de plusieurs comme pour la figure 5, les expériences à venir devant montrer lequel des deux procédés était le meilleur, La révélation statistique d'un faisceau de lignes parallèles est peut-être due à la façon dont procédait GILBERT pour rendre le matériau « uniforme »; il le faisait passer par des tamis de mailles voisines. Si c'est la vitesse de sédimentation qui constitue le critère dynamique dont dépend le transport plutôt que les dimensions des particules, Ie matériau plus fin, dont la vitesse de sédimentation varie sensiblement comme le carré des dimensions, sera en effet dispersé trois fois plus vite que les matériaux plus gros dont la vitesse de sédimentation varie comme la racine carrée des dimensions, en supposant que les tamis sont entre eux dans un rapport déterminé; le peu d'expérience qu'on possède à ce sujet montre que la dispersion tend à élever les lignes.

Si $b / d$ et $\mathrm{D} / d$ constituaient des facteurs liés a la définition du coefficient de fond pour le domaine étudié, la dispersion des points empêchait de les différencier.

On peut arriver donc aux conclusions suivantes pour des matériaux uniformes :

a) Le coefficient de fond est un critère valable en ce qui concerne le charriage de fond dans toutes les phases de ce mouvement;

b) Ce coefficient est fonction du calibre des grains et de la charge;

c) Les résultats dont on dispose présentent des lacunes empêchant la définition exacte de la relation fonctionnelle existant entre le facteur de fond, le calibre des grains et 
la charge. Cette relation peul etre simple de forme, mais discontinue comme sur la figure 1 , ou un peu plus compliquée, mais continue, comme sur la figure 5. Les courbes de ces deux figures sont également admissibles entre les limites imposées par les considérations statistiques.

2. Coefficient de Fond. - Cas des sables Naturers. - Une première étude de la granulométrie d'échantillons de sable de rivière présumé naturel a confirmé ce que les sédimentologues paraissent savoir depuis longtemps et que les ingénieurs se sont mis à redécouvrir récemment [14], à savoir que ces sables obéissent à la loi classique desi erreurs par le logarithme de la dimension des grains, la fréquence étant évaluée d'après le poids. On a découvert ainsi le fait suivant, intéressant au point de vue cuantitatif :

L'écart-type du log. de la dimension $=2,6$ $X$ (vitesse de sédimentation du grain moyen $)^{5 / 12}$.

On sait très bien que la dispersion augmente avec la grosseur moyenne des matériaux naturels. La forme que prend l'équation de la dispersion pour les sables ne semble pas avoir de signification physique particulière; elle est valable depuis les vitesses de $0,8 \mathrm{~cm} / \mathrm{s}$ jusqu'à $35 \mathrm{~cm} / \mathrm{s}$. Les graviers donnaient, en général, sur un papier gradué en probabilité logarithmique, des droites incurvées avec un caractère familial commun bien marqué. Il a été décidé que la ligne de séparation entre graviers et sable pouvait être fixée à peu près à $1 \mathrm{~mm}$; indisculablement, elle correspond en réalité au point où la vitesse de sédimentation cesse d'être proportionnelle à $\mathrm{D}$ pour devenir proportionnelle à sa racine.

L'étude a permis d'affirmer qu'une grosseul. moyenne de grain pouvait parfaitement servir de critere pour les sables, mais il n'a pas été possible de déterminer une grosseur moyenne valable pour les graviers. II a été également reconnu que les sables USWES, qui sortent des limites quelque peu arbitraires de la concordance avec la probabilité de répartition logarithmique, devaient être écartés de l'analyse suivant la thérie du régime.

Les valeurs de Srraub ont été traitées exactement comme celles de Grubert et portées sur la figure 5. Ceux des sables USWES qui avaient été acceptés devaient être soumis à un traitement spécial, parce qu'ils étaient destinés à des essais durant chacun à peu près une heure; et l'expérience a montré que, dans des expériences de ce genre, on approche du régime asymptotiquement el que, par conséquent, des durées de l'or- dre d'un jour ou deux sont nécessaires pour obtenir les profondeurs finales avec une charge et un débit donnés. Nous avons estimé que lorsque les expériences semblaient être arrivées à une limite, les profondeurs étaient probablement $85 \%$ à $90 \%$ des profondeurs finales. Rien que pour trouver la relation fonctionnelle existant avec C, il serait donc bon de calculer l'écart relatif du coefficient de fond en fonction de la valeur de $\mathrm{F}_{b 0}$, elle-même trouvée par extrapolation d'après un diagramme de $F_{b}$ en fonction de $C$, les erreurs de profondeur étant ainsi éliminées. C'est ce qui a été fait, et le résultat obtenu est reproduit sur la figure 5. Pratiquement, tous les points correspondaient à un régime de dunes.

On pourrait done tirer les conclusions suivantes pour les sables naturels de rivière en régime de dunes (voire mème au-delà, si l'on voulait extrapoler) :

a) Je facteur de fond constitue un critère valable pour le charriage de fond;

b) le calibre du matériau de fond peut être représenté par le calibre moyen des grains;

c) le facteur de fond est fonction du calibre des grains et de la charge;

d) la formule fonctionnelle est probablement la même que pour les matériaux uniformes, sauf peut-être en ce qui concerne $D$ qui doit prendre en compte le coefficient de dispersion du grain; les constantes diffèrent probablement.

3. Eouation de la pente de RÉgrme. Sables UNIFORMES ET NATURELs. - Lors de l'analyse préalable, le premier test portait sur toutes les observations. Autrement dit, on portait sur du papier quadrillé ordinaire :

$$
\mathrm{K}=\mathrm{V}^{2} / g d \mathrm{~S} \text { divisé par }(\mathrm{V} b / \nu)^{0,25}
$$

en fonction de C. Dans l'analyse préliminaire, le domaine de $\mathrm{C}$ correspondant à la structure en dunes était très réduit et le diagramme révélait une relation linéaire. En seconde analyse, l'introduction des observations sur le régime à antidunes reléguait la zone des dunes dans l'angle de gauche de la feuille, si bien que les points portés semblaient vides de sens (voir fig. 6). Cependant, quand on eut tracé la courbe subcritique de l'équation ( $3 a$ ) sur les relevés correspondant à tous les matériaux, et qu'on eut tiré le meilleur profit des points terriblement dispersés de la zone supercritique, la figure 7 présenta une image de ce que l'on pourrait attendre d'expériences à grande échelle savamment menées. A noter que les seules observations concernant le sable naturel sont celles de STraub; 


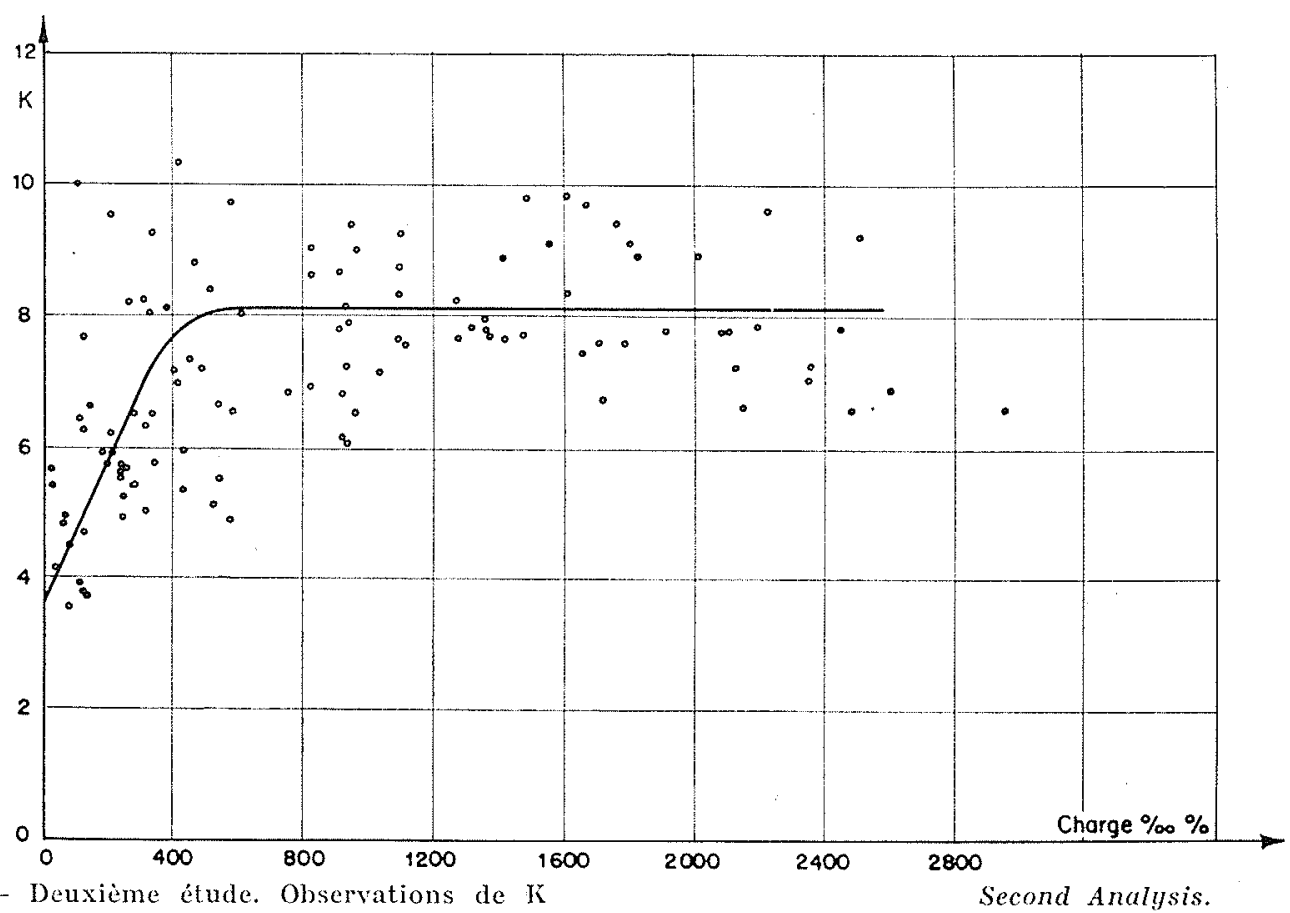

Fic. 6. - Deuxieme étude Observations de $K$ pour un sable de Gilbert, en tous regimes.

celles du Corps du Génie ne contiennent pas les pentes.

On a donc pu conclure :

a) En ce qui concerne les dunes, que l'équation de la pente de régime est : $\mathrm{V} \cdot / g d \mathrm{~S}=3,63(1+a \mathrm{C})(\mathrm{V} b / \nu)^{0,25}$

$a$ valant environ $1 / 400$ pour les matériaux uniformes et ayant probablement une autre valeur pour les matériaux naturels de rivière;

b) lors du passage du régime des dunes à celui du fond lisse, l'équation (3) fait apparaître une transition, l'état final semblant ètre tel que $1+a \mathrm{C}$ prend une valeur déterminée, dépendant de la grosseur du matériau;

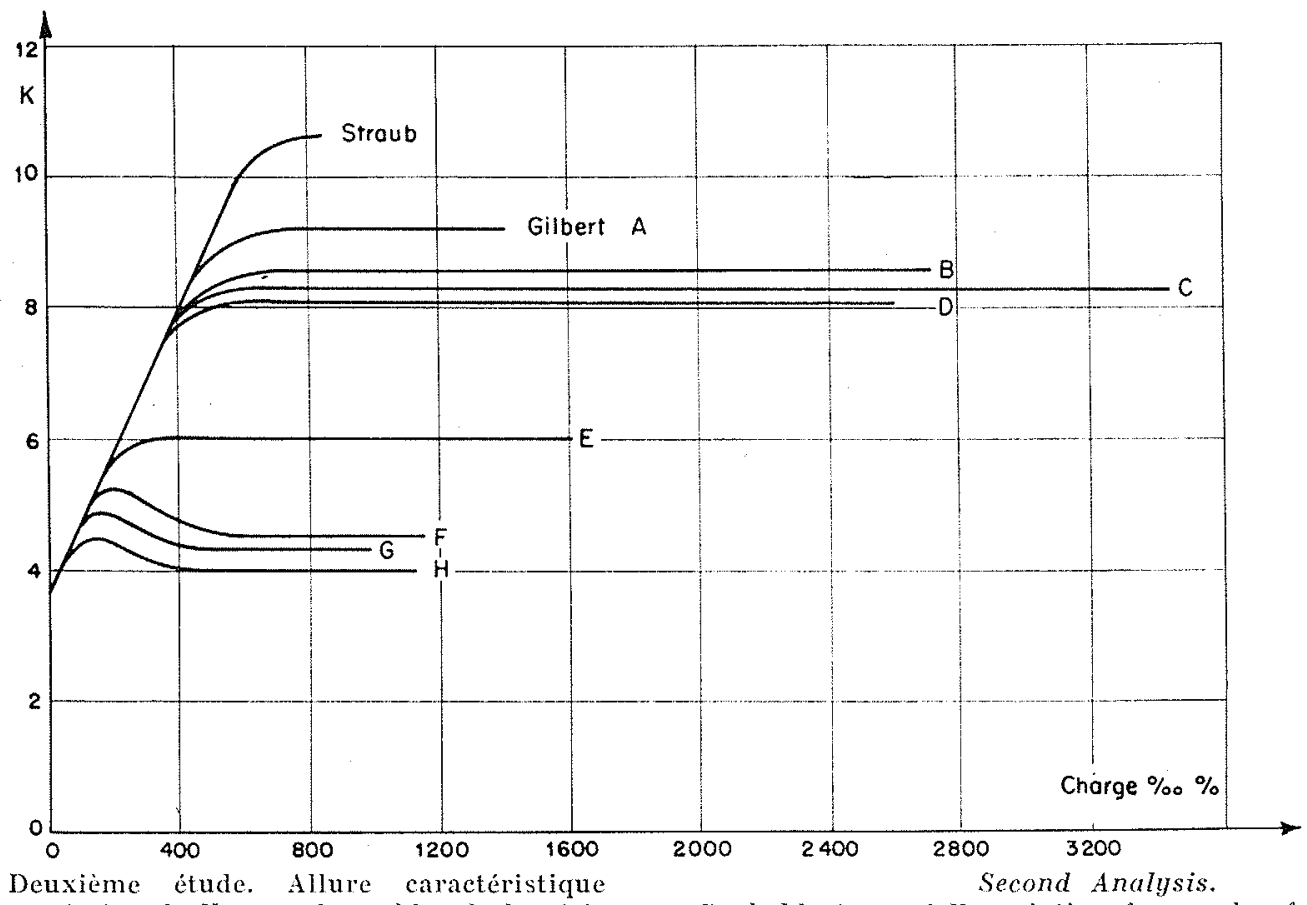

Fra. 7. - Deuxième étude. Allure caractéristique probable de la variation de $K$ pour des sables de la série

lrobable type of $K$ variation for sands of Gilbert range. de Gilípert. 
c) les observations relatives aux phases supercritiques sont si dispersées qu'il n'est pas possible de rien affirmer au sujet des valeurs numériques des constantes.

\section{Analyse dimensionnelle}

L'analyse dimensionnelle, appliquée aux problèmes relativement faciles posés par l'hydraulique des parois rigides, n'utilise que quelquesuns des différents groupes non dimensionnels possibles. Avec des parois mobiles, il convient d'abord d'établir les formules que permet raisonnablement d'admettre la théorie du régime, en tenant compte des diagrammes tracés d'après les résultats réels obtenus, puis de reconnaître au moyen de l'analyse dimensionnelle le degré de généralisation qu'elles admettent.

Adoptant ce procédé, nous pouvons affirmer que si un canal artificiel de largeur $b$ est établi dans un champ gravitationnel $g$ et si l'on y établit un débit $Q$ d'un fluide de masse spécifique of et de viscosité cinématique $v$, portant une charge $C$ de matériau uniforme de diamètre D et de masse spécifique $o_{*}$, on constatera qu'un certain régime se manifeste de lui-même avec une profondeur $d$, un gradient d'énergie $g \mathrm{~S}$, une répartition d'efforts de frottement $\tau$ sur les parois limites et une vitesse moyenne V. En langage mathématique abrégé, on dira:

$d, g \mathrm{~S}, \tau, \mathrm{V}=$ fonctions de $\left(b, \mathrm{C}, \mathrm{D}, \mathrm{Q}, g, v, \mathrm{p}_{s}, \mathrm{p}_{\mathrm{i}}\right)$

Physiquement parlant, nous qualifierons de «liées » les quatre variables qui figurent au premier membre et d' "indépendantes » les huit variables du second; le mathématicien est d'ailleurs tout disposé à choisir quatre variables quelconques pour les placer à gauche et mettre à droite les huit restantes. Les trois unités fondamentales de masse, longueur et temps étant contenues dans toutes les variables, on pourra remplacer les équations précédentes par autant d'équations entre des variables nouvelles constituées de groupes non dimensionnels; on aura ainsi huit variables indépendantes moins trois, c'est-à-dire cinq. Dans le réel, il peut y en avoir moins de cinq, mais le calcul dimensionnel n'admet pas qu'il puisse $\mathrm{y}$ en avoir davantage.

Le lecteur pourra vérifier que les équations ci-après satisfont entièrement aux exigences du calcul dimensionnel :

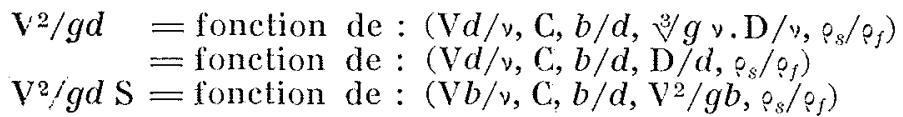

(Pour cette vérification, il $y$ a lieu de voir si le premier membre est une fonction sans dimensions d'une ou de deux variables liées, si le second membre contient cing groupes dont cha- cun contient un terme manquant dans les autres et si toutes les variables indépendantes ont été utilisées.)

Les équations (X), (Y) représentent des généralisations possibles des équations (5) à (7) au sens dimensionnel du mot, bien que pratiquement elles puissent constituer des généralisations excessives; l'équation $(Z)$ correspond à l'équation (3). Toutes les équations obtenues par l'analyse peuvent, manifestement, dépendre de la densité relative, ce qui est vrai sans aucun doute pour celles du facteur de fond et probable pour celle qui exprime la pente; sur le terrain, cette grandeur ne varie pas, non plus que dans les essais qui ont conduit aux résultats expérimentaux; aussi pouvons-nous ne plus en tenir compte. Il semble également que les équations donnant le facteur de fond et la pente peuvent dépendre du rapport $b / d$; physiquement parlant, ceci est hors de doute, mais cette influence n'est pas suffisamment importante pour se dégager de la gamme correspondant aux résultats du tableau I. Il est intéressant de noter que $\mathrm{Vd} / \nu$ peut intervenir dans les formules du facteur de fond; que l'analyse ne l'ait pas mis en évidence n'a rien d'étonnant, si l'on songe au rôla effacé que joue son équivalent dans toute une série d'expériences sur conduites rigides rugueuses. Ce n'en est pas moins un avertissement sur l'importance qu'il peut prendre aux faibles valeurs, inférieures à celles qui caractérisent les observations considérées.

L'équation (Y) établit que l'expression donnant $F_{b 0}$ et qui dépend de $D_{\text {p }}$ peut devenir fonction de $\mathrm{D} / d$ pour de grandes valeurs de ce rapport; elle pose également la question de savoir si cette relation existerait dans le domaine supercritique, mais aurait échappé à l'attention à cause des faibles variations de la petite valeur de $d$ dans ce domaine. Nous avons déjà eu l'occasion de reconnaître que les résultats obtenus ne prouvent à peu près rien quant à la forme exacte de l'influence de $D$, même dans le domaine subcritique. Le terme $\mathrm{V}^{2} / g b$ qui figure dans $(Z)$ peut être insignifiant au point de vue physique, si on est assez fondé pour imaginer qu'il mesure en quelque sorte l'équilibre qui intervient entre la poussée ascendante due à la turbulence engendrée près du fond d'une part, et le poids des particules qui le forment, d'autre part; il n'y a pas de matériau ascendant sur les parois comme il y en a sur le fond. Enfin, le terme caractéristique $(\nu g)^{1 / 3} . \mathrm{D} /$ y signifie probablement qu'il conviendrait mieux, au point de vue dynamique, de considérer la vitesse de sédimentation que le calibre du grain.

Dans l'ensemble, rien ne prouve que les formules ne conviennent pas pour la gamme des observations qu'elles interprètent, mais il existe de bonnes indications permettant de justifier 
leur validité ou de délimiter leur domaine de validité lorsque l'on sort de la gamme des observations recueillies ou que l'on se trouve à sa limite.

\section{Recherches collectives}

Si l'on veut s'affranchir des lacunes révélées par les pages précédentes, une recherche collective serait néeessaire, confiée à des organismes possédant des moyens financiers suffisants et capables de mettre en ouvre de grands débits. (Il y en a qui pourraient faire dévier des débits de plusieurs centaines de $\mathrm{m}^{3} / \mathrm{s}$ des canaux et les y ramener sans grande peine.) Pratiquement, il s'agirait en première urgence d'établir, pour les matériaux de fond des canaux naturels, en eau claire et à une température standard, la relation liant d'une part le paramètre $K$ qui entre dans la formule du facteur de fond et dans celle de la pente de régime, d'autre part le calibre du matériau de fond et la charge du charriage de fond, ceci dans les trois phases du mouvement (dunes, fond lisse, antidunes), mais plus particulièrement dans la première. On pourrait, dans une certaine mesure, effectuer simultanément les recherches fondamentales conduisant à l'ensemble des objectifs poursuivis. Ainsi, il n'y' aura pas lieu de commencer par rechercher la longueur minimum donnant des conditions uniformes et une pente mesurable, si l'on travaille sur des canaux naturels ou artificiels ayant manifestement la longueur convenable; d'autre part, on ne devra pas procéder à des essais sur des graviers naturels tant que des observations nombreuses effectuées sur le terrain (et qui nécessiteraient probablement l'appui des services de la voirie, des exploitants de gravières et des géomorphologues) n'auraient pas dévoilé le mécanisme de l'élaboration des graviers naturels formant la partie active des fonds de rivière. Une conférence internationale, ou la réunion de plusieurs conférences de caractère national [18] pourraient être convoquées pour essayer de répartir la besogne. I.es points principaux sur lesquels la recherche aurait à porter seraient :

1. -. La longueur du canal expérimental et la durée d'observation permettant de réaliser :

a) un régime permanent de charriage de fond; b) la mesure précise d'une pente uniforme.

2. - Le mécanisme d'élaboration des graviers naturels actifs dans le lit d'une rivière.

3. - Détermination de la relation, entre : d'une part le paramètre caractéristique du facteur de fond et de la pente de régime, d'autre part le calibre et la charge du matériau de fond; ceci pour un charriage de fond par de l'eau claire à une température standard, avec des débits atteignant $15 \mathrm{~m}^{3} / \mathrm{s}$, en régimes à dunes, à fond lisse et à antidunes; l'attention devrait porter surtout sur les points antérieurement négligés :

a) les sables naturellement triés de toutes dimensions,

b) les matériaux plus fins que $0,3 \mathrm{~mm}$ et allant jusqu'à $0,1 \mathrm{~mm}$,

c) les matériaux compris entre $7 \mathrm{~mm}$ et environ $20 \mathrm{~kg}$

d) les graviers naturellement triés,

e) les calibres suffisamment uniformes, inférieurs à $0,4 \mathrm{~mm}$,

$f$ ) les charges comprises entre des valeurs très faibles et environ 30 cent-millièmes (en poids)

g) les transitions d'une phase à une autre,

h) Ies matériaux de fond ayant des dimensions appréciables par rapport à la profondeur, comme e'est le cas pour l'écoulement supercritique sur des graviers.

4. - Influence de la viscosité du fluide sur les relations trouvées.

5. - Influence de la charge en suspension sur la viscosité apparente.

6. - Comportement de certains matériaux particulièrement uniformes, tels que le verre ou les dodécaèdres en matières plastiques, n'accusant pas les différences de forme, ou de densité, propres aux matériaux naturels.

7. - Effel de la densité relative du matériau transporté et du fluide.

Tous les résultats d'observation devraient être publiés sous leur forme originale.

(La Bibliographie se trouve à la fin du texte anglais. p '157) 


\title{
Regime analysis of laboratory data on bed-load transport
}

\author{
B Y \\ T. BLENCH ${ }^{(1)}$ \\ AND \\ R. BRYAN ERB ${ }^{(2)}$ \\ n. SC., M.I.C.E., M.A.S.C.E. \\ I. $\mathrm{Sc}$.
}

(See French text p. 132 for illustration)

\begin{abstract}
The nature, derivation and, particularly, the practical use of regime theory $[1,2,3,10,11]$ in canal design are outlined briefly, to prepare the ground for the sequel. Then the methods of the theory are applied to analyse available data of transport of sands and fine gravels in
\end{abstract}

\begin{abstract}
laboratory flumes. Simple and novel results are obtained, regine theory receives some generalisation, and the data are shown to be inadequate to cover the field of practical interest. Suggestions are made for international cooperative research.
\end{abstract}

\section{INTRODUCTION}

There are two major oppositely directed approaches to the bed-load transport problem. The regime theory approach $[1,2,3,10,11]$ originated in India at the end of the nineteenth century and gave formulas acceptable to the Government of India for design purposes in 1933 13]. The formulas fit into the framework of rigid-boundary hydraulics and also generalise that subject. They were derived by studying the interrelation, through velocity, of the breadth, depth and slope of channels that had adjusted themselves to flnal equilibrium (regime) values despite the futile efforts of engineers to force them to run unnaturally. Because of their origin they define factors related to the bed-load and to the cohesiveness of the sides in terms of $\mathrm{V}, b, d$ and ignore the physical properties of the bed-load and side material that cause factors to exist; in this respect they are analogous to the equations of electromagnetism that define "quantities" of electricity and magnetism by their results instead of by their imperfectly understood causes. Mathematically one might say that the regime equations are what would be obtained from sediment transport equations if the sediment para-

(*) Professor of Civil Engineering, University of Alberta, Canada, and Consulting Engineer.

(*) Research Engineer, A.V. Roe Co., Toronto, Canada. meters were eliminated and, of course, if the sediment transport equations happened to be known. The laboratory approach, as it will be called here, has developed in Europe and the U.S.A. through the study of sediment-bearing flow in laboratory flumes, because the vast systems of regularly observed, selfadjusting canals needed for field study did not exist there. The many formulas produced have tried to relate the flow properties observeable in such flumes to the measureable characteristics of the bed-load, and there have been attemps to study suspended load that are not relevant to this article. The formulas that seem to have the most dynamical foundation $[7-9]$ are presented, nowadays, with such complexity that few engineers can understand their derivation or use, or visualise the range of data from which they came; moreover they do not relate the original data, but quantities derived from them by assuming uniform flow over a channel section and then deducing the part of the channel section that may be "attributed to the bed" if Manning"s equation applies.

The authors feel that, in principle, both methods of approach are legitimate and should be extended till they can be reconciled. Their own constructive step towards this desireable objective has been to use the methods of regime theory to analyse the classic data of GrLbert [4], that seem to form the 
core of the Meyer-Peter and Ersstem information [7-9], and some more recent data on transport of natural river-bed sands in flumes $[16,17]$. The labor was great, but the methods are exceedingly simple and the results can be comprehended readily. Perhaps the greatest gain from the work is the discovery that present laboratory data are utterly inadequate to produce formulas for the practical designer; this discovery points the way to future rescarch.

Accordingly the authors outline their ideas and results. Only an outline is possible within reasonable space, but the references will give the details needed by an intending investigator. As the idea of regime theory are basic the next three Sections will outline this subject; they are copied almost verbatim from Bib. 10, by permission of the A.S.C.E. The reader acquainted with previous writings on the subject will note that the nomenclature has been changed to suit A.S.C.E. practice, and that a result of the present article has been anticipated in the slope formula.

\section{General conditions for exact applicability of regime equations}

Regime theory equations were devised, in dynamically acceptable forms, to fit the correlations observed amongst the measureable variables of artificial channels that moved a noncohesive bedload in dune formation, had cohesive erodible sides, and had adjusted breadth, depth and slope to final values, at which they are said to be "in regime". It is concluded that the equations would be exactly applicable to channels :

i. That are straight.

ii. Whose sides behave as if hydraulically "smooth". (Technically, this means that the roughness constant of the sides depends only on the nature of the fluid, however complex.)

iii. Whose bed-width exceeds three times the depth. iv. Whose sides stand at slopes approximating those found for cohesive sides in nature.

v. Whose discharges are steady.

vi. Whose sediment-load, however complex, is steady.

vii. That move a non-cohesive load, however small, along their bed in dune formation.

viii. That run at speed less than critical.

$i x$. That have adjusted their width, depth and slope to final (or regime) values. (The implication here is that, except by the remotest chance, a channel of the type contemplated will not be designed to carry its sediment-load and discharge without some addition to or removal from its boundaries; therefore self-adjustments will occur till regime is attained.)

\section{Conditions for practical applicability of regime equations}

Experience, and attention to dynamical principles, permit the extension of resime theory equa- tions well beyond the limits of exact applicability, even to heavily charged rivers and tidal estuaries $[5,6,10]$. For artificial channels that might reasonably be classed as canals, the following comments on the numbered points of the preceding section should indicate the amount of relaxation that may be permitted in using the equations for most practical problems. The numbers below correspond to the same ones of the preceding Section.

$i$. The curvature of ordinary channels may be neglected. Sections on curves will deepen on the side away from the center of curvature and shoal on the other, but it is not usual to excavate to anticipate this.

ii. The author bas never found the assumption of smooth sides to result in unsuitable designs.

iii. The limit of bed-width to depth ratio has been fixed because of lack of experience of any thing smaller, and not because it is known to be a true limit.

iv. Nature usually adjusts cohesive sides to slopes steeper than 1 upon 1; but designers sometimes make them to 1 upon 2 or 3 . Probably the shear stress on such flat sides intensifies at the base, so that the equation that gives side shear as constant all over is violated locally.

v. Designed full supply discharge usually suffices in formulas in practice, since the bed ceases to be active at discharges slightly below this value unless the channel carries a most abnormal bed-load. The latter exception may be covered by using the longterm mean probable discharge in excess of that at which bed-movement is likely to just start.

$v i$. Except in head-reaches the most violent fluctuations of sediment-load, however complex, have no effect that will embarrass the engineer running the channels. A small sea. sonal fluctuation of channels about their mean regime condition is to be expected; so is a series of secular oscillations. Design to a mean-load condition is satisfactory.

vii. The physical separation between absolutely no bed-movement, and the very least, is analogous to that between non-turbulent and turbulent flow. The very least bed-movement will result in dunes developing; the friction coefficient will then change completely to correspond to dune-formation and the regime equations will apply. With absolutely no movement at any time, regime equations do not apply. If in doubt, movement should be assumed possible.

viii. It is not implied that dune formation ceases suddenly exactly at critical velocity calculated from mean depth. The scanty and poor evidence of flume flow near critical suggests that this critical velocity is probably a top limit beyond which dunes will not be found. The scatter of flume data, combined with information from rivers and models, suggest that there is actually a considerable "transition zone ", mainly below critical, in which the presence or absence of dunes is decided 
by small disturbances. Fortunately the practical canal designer is unlikely to be interested in channels running near critical velocity.

$i x$. Two of the regime equations can be interpreted as sediment-transport formulas and used accordingly for temporarily adjusted conditions. However, discussion of this is outside the scope of the present Paper.

\section{The three basic formulas of regime theory}

The three practical design formulas of regime theory are in forms that hide their meanings; but an understanding of natural channel self-adjustments and the laws behind them is essential for practical design. Therefore, this Section discusses the three basic formulas from which the practical ones have been derived. (The various symbols are defined briefly in Appendix $\mathrm{I}, \mathrm{p}, 156$, and discussed more fully in the text, as necessary.

The deduction that three independent basic equations must exist follows from the observed consequences of excavating, to arbitrary dimensions in an erodible material, a channel that carries a prescribed discharge of a prescribed fluid with a prescribed sediment-load, however complex. The breadth, depth and slope will adjust to values at which the load can! be carried without, on an average, any deposition on or removal from bed or sides. This self-adjustment is in accordance with Jaws of sediment transport so that, given sufficient knowledge, $b, d, \quad S\left(^{*}\right)$ should be expressible in terms of the discharge and the parameters that define the nature of the water-sediment complex (as the mixture of fluid, suspended, and bed loads is called). Therefore there should be interrelationships amongst $b, d, \mathrm{~S}$ and other measureable consequences of the set-up, e.g. mean velocity $V$. These relations should provide a means for defining watersediment properties, just as relations amongst electric and magnetic forces provide a means for defining "quantities" of electricity and magnetism.

The three dynamically satisfying relationships. found from channels that have adjusted their $b, d, s$ to equilibrium values - and are then said to be "in regime" - are conveniently discussed in the forms given below. (It would be permissible, of course, to use any other three independent ones obtained by algebraic manipulation.)

\section{i. A bed-factor $F_{b}$ may be defined by :}

$$
\mathrm{V}^{2} / d=\mathrm{F}_{b}
$$

which arises from the observation that $\mathrm{V}^{2} / d$ remains fixed throughout a channel system in which the constitution of the water-sediment complex remains practically unaltered.

The measurement of $d$ needs some discussion. A natural straight channel with erodible cohesive sides assumes a form that may be considered as a trapezoid whose bed is traversed by moving dunes. The accurate measurement of a mean depth $d$, in an actual channel, would require the observation of

$\left.{ }^{*}\right) b, d \mathrm{~S}=$ breadth, depth, slope. cross-sectional profiles covering several dune lengths and made at space intervals that would avoid bias in favour of either troughs or crests; alternatively profiles could be observed many times at one place. In the laboratory the former method is common; in the field, the engineer engaged in discharge observations tends to adopt the latter somewhat unconsciously by taking a few sections at one place for each periodic rating curve and noticing that he does not have to alter mean bedlevel very much for subsequent ratings. For construction the channel is excavated to the depth calculated as $d$, and the actual depth that imposes itseif eventually should not differ enough to cause notice, if the design has been a good anticipation.

The mean velocity $V$ is that found from discharge $Q$, and the cross-sectional area of the trapezoid of depth $d$ from water-surface to mean bed.

For many practical purposes $d$ may be found from one careful sectional observation .

ii. A side-factor $\mathrm{F}_{\mathrm{s}}$ may be defined by :

$$
\mathrm{V}^{3} / b=\mathrm{F}_{\mathrm{s}}
$$

where $b$ is defined, for convenience, as the breadth that, multiplied by $d$, gives the area of the mean trapezoidal section; practically it may be taken as breadth at half depth. This formula came from manipulation of others, and not from direct observation; direct observation would not have been suitable because the nature of the sides varies greatly from place to place.

If $F_{s}$ is multiplied by the square of the mass density and by the kinematic viscosity the result has the dimensions of the square of stress intensity. The stress intensity represented is believed to be a measure of the shear stress intensity on the sides, provided that the sides are hydranlically " smooth". That the sides of artificial canals in fair maintenance should be " smooth", when the material of which they are made might be classed as "rough" in the laboratory, may seem slighty paradoxical. The paradox vanishes when it is remembered that socalled smoothness depends not on roughness height, but on relative roughness height, and the relative height is very much less in the field than in the laboratory; even large concrete tunnels will be found to work in the near-smooth stage whereas concrete pipe of usual works in the rough stage.

iii The flow formula is a generalization of that of Brasius for smooth rigid-boundary pipes, and is :

$$
\mathrm{V}^{2} / g d \mathrm{~S}=3.63(1+a \mathrm{C}) .(\mathrm{V} b / v)^{0.25}
$$

where $a \mathrm{C}$ is likely to be negligible in most practical artificial channels. That a " smooth" boundary formula should be correct is obvious enough once it has been discovered. The unfortunate term "smooth", in rigid-boundary hydraulies, means only that the fluid near the boundary exists in a particular phase of motion which permits the flow resistance to be calculated from the fluid properties - - viscosity in that case - while the solid boundary: acts only as an anchor. In a regime channel the bed and zone near it are differentiated out of the water-sediment complex.

The term $(1+a \mathrm{C})$ has been added recently by 
the writer for reasons detailed elsewhere [12]. The original formula without the term was found by C. KING [15] from irrigation canal data where $C$ was too small to be noticeable. The value of " $a$ " is about $1 / 400$ for uniform sand such as used in the classic GILizenT flume experiments [4]; lack of data prevents the writer from stating a definite value for natural sands and gravels, but he recommends $1 / 233$ tentatively. $\mathrm{G}$ is measured in thousandths of one percent by weight and is the ratio of weight (in air) of bed-load per unit time to weight of water per unit time. It could be called a concentration, were it not that that term suggests a uniform distribution; therefore it is given the special name "charge".

\section{The three basic design formulas of regime theory}

The typical problem of practical channel design may be stated as

"Given discharge, the nature of the water-sediment complex as expressed by linematic viscosity and bed-factor, and the susceptibility of the sides to erosion as expressed by side-factor. Find the breadth, depth, and slope at which the channel can run in regime."

To solve it without unnecessary algebra, equations (1) to (3) must be manipulated to give $b, d$ and $S$ directly in terms of $Q, F_{b}$, and $F_{s}$. The most rapid way to transform equations (1) to (3) is to treat (1) and (2) as follows: whence :

$$
\left(\mathrm{V}^{2} / d\right) \cdot\left(\mathrm{V}^{3} / b\right) \cdot \mathrm{V} / \mathrm{V}=\mathrm{V}^{6} / \mathrm{Q}=\mathrm{F}_{b} \cdot \mathrm{F}_{s}
$$

$$
\mathrm{V}=\left(\mathrm{F}_{b} \cdot \mathrm{F}_{s} \cdot \mathrm{Q}\right)^{1 / 6}
$$

Then insert this value of $\mathrm{V}$ into the three basic equations to obtain :

$$
\begin{aligned}
& b=\left(\mathrm{F}_{b} \mathrm{Q} / \mathrm{F}_{s}\right)^{1 / 2} \\
& d=\left(\mathrm{F}_{s} \mathrm{Q} / \mathrm{F}_{b}{ }^{2}\right)^{1 / 3} \\
& \mathrm{~S}=\frac{\mathrm{F}_{b^{5 / 6}} \mathrm{~F}_{s}^{1 / 2} y^{1 / 4}}{3.63(1+a \mathrm{C}) g \mathrm{Q}^{1 / 6}}
\end{aligned}
$$

in which " $a$ " is very poorly known, but is recommended tentatively as $1 / 233$, and the whole term $(1+a \mathrm{C})$ is unlikely to differ appreciably from 1.0 except in channels with very abnormal loads.

Equations (A) (B) (C) are the basic design formulas of regime theory. Means of estimating $F_{b}, F_{s}$ are discussed elsewhere $(1,2,10)$.

\section{Data of regime theory and laboratory compared}

Table $1\left(^{*}\right)$ compares the ranges of some important variables relevant to regime theory and the laboratory approach respectively, under the headings "Canals " and "Gilbert, Meyer-Peter and EissTerN"; information on experiments in the laboratory with natural river sands are not included but would not extend the comparison except as to "grading". Attention is drawn to the following features:

* Cf. p. 157 . $i$. The ranges of data of the two approaches are largely complementary. Thus, canals cover two cycles of ten of the very significant Reynold's Number in terms of width while flumes cover one adjacent cycle. Canals have the enormous discharge range from 1 to 10,000 cusecs, while flumes have from 0.1 to 1.0 cusecs. Canals have only dunes, but flumes have also sheet flow and antidunes. Canal bed load charges are trifling and not even measured, but flume charges run up to 3,000 parts per hundred thousand by weight, which would occur in nature under only the most unusual circumstances. The canal bed-factor (which is just a proportional to Froude Number in terms of depth) ranges from 0.5 to 1.5 ; but the flume range is from 1 to 150 .

ii. There is a common factor of smooth sides, whose absence would make combination of the data awkward it not impossible.

iii. There is a common deficiency in regard to particle size. From the viewpoint of a river engineer designing works in gravel or boulder streams with material from, say, $25 \mathrm{~mm}$ to $300 \mathrm{~mm}$ size, the canal range of 0.1 to $0.60 \mathrm{~mm}$ and the flume range of 0.3 to $7.0 \mathrm{~mm}$ with one freak of $28.6 \mathrm{~mm}$ (for which the authors have failed to obtain the original hydraulic data) are comparable and defective.

iv. The laboratory data for uniform materials may yield correct functional forms but cannot give correct coefficients for natural river-bed sands.

These features lead to some interesting reflections. First, different types of formulas obtained from the two sets of data can hardly be expected to agree perfectly with each other when extrapolated into each other's domains. Second, there is urgent need to try to fit both ranges by either new formulas or existing ones modified so as not to lose their dynamical sense. Third, even if both ranges can be fitted by the one set of formulas the deficiency in respect to particle size will leave considerable doubt on the applicability to channels carrying gravel or boulders; so there is a need for more research. This last reflection draws attention to a peculiarity of the Meyer-Peter formula, that it purports to tell how much material is being transported per unit width of a channel if the discharge intensity, the slope, and the particle size are given, and to apply equally well for 6 inch material in a river channel 30 feet deep as to 6 inch material in a channel 1 foot deep; there seems nothing intrinsically absurd in this proposition, but it is remarkable enough to merit verification from material larger than $7.0 \mathrm{~mm}$.

\section{Preliminary regime analysis of Gilbert data (12)}

For regime analysis the Gilisent data [4] were a round thousandi " regime canal observations" with the usual smooth sides. But they had the advantage of measured bed material sizes, $D$, and bed-load charges, $\mathrm{C},(\mathrm{C}$ is the ratio of weight of bedload per unit time to weight of water per mit time, expressed in thousandths of one percent or parts per hundred thousand), and they called for 
no investigation of "side factor" [equation (2)] since sides were not self-adjusting. So preliminary regime investigation aimed at finding whether :

$i$. The bed-factor, $\mathrm{F}_{b}=\mathrm{V} \% / d$, can be represented as a function of $C$ and $D$, without prejudice to the possibility that $b / d, \mathrm{D} / d$ and viscosity might also be relevant.

ii. The constant 3.63 in the regime slope formula discovered from field canals, whose $\mathrm{C}$ is very small, viz:

$$
\mathrm{V} 2 / g d \mathrm{~S}=3.63(\mathrm{~V} b / \mathrm{v})^{0.25}
$$

needs adjustment in terms of $\mathrm{C}$, without prejudice to the possibility that $b / d$ and $\mathrm{D} / d$ might be relevant.

\section{INVESTIGATION OF ITEM I}

An initial rapid check of the probable existence of a bed-factor relation was made possible by a table of data, for one size of material, purportins to be for $(a)$ the change of phase from dunes to sheet flow $(b)$ the change of phase from sheet flow to antidunes. It seemed likely that a change of phase would oceur at a definite Froude Number or, what is the same, bed-factor. If it did, then it would also be expected to oceur at a definite beclload charge irrespective of the discharge or total load. Accordingly, for each change point, or critical point - to use a GILBERT term - $\mathrm{V}$ was plotted against $d$, and load against discharge. The first plot gave a square root relation in both cases; the second gave a proportionality relation in both cases. This confirmed that bed-factor was a good criterion for change of phase right outside the conditions studied in field canals, and that charge was a principal variable in the functional expression for bed-factor.

With encouragement from the preceding results the next step was to start plotting, on ordinary squared paper, the bed-factors for all the Grlbert materials, A- $\mathrm{H}$, (sizes $0.30-7.0 \mathrm{~mm}$ ) against charge, using distinctive marks for each material. The fitting lines of the finally accepted diagram are reproduced in Fig. 1. They represent one reasonable way to fit the data and are valuable as a step to further analysis and as an indication of inadequacies of existing data for testing any hypothesis whatever. To ensure that their status will not be misunderstood the history of their development is outlined below in some detail.

As plotting proceeded the nature of the data started to become apparent. Scatter was, on the whole, as wide as would be expected from field canal data. In the dune zone it was worse than in that of sheet flow, and in the antidune zone it looked so bad that antidune points were dropped from the analysis. Data for some materials were very scanty in the dune zone and, for all materials, were inadequate, in the region of small charge, to permit any useful assessment of the likely value of $F_{b 0}=$ value of $F_{b}$ for charge tending to zero. This was unfortunate since designers of canals are most interested in $\mathrm{F}_{b 0}$, and theoretical investigators like to have the start of a fitting curve defined if possible. However, practical canal designers know that, for the sand range with trilling charges, a working approximation to $\mathrm{F}_{b 0}$ is :

$$
F_{b 0}=1.9 . D_{m}^{0.5} \quad \text { (4) Empirical }
$$

where $D_{m}$ is the median size of bed-material in $\mathrm{mm}$, and $\mathrm{F}_{b 0}$ is, as usual, in $\mathrm{ft} / \mathrm{sec}^{2}$. A practical designer would hesitate to use this for material as large as $7 \mathrm{~mm}$, but a glance at Fig. 1 will show that a $50 \%$ error would hardly make any difference to conclusions on functional form of the charge relation. Therefore, this formula was used for fixing the likely positions of the bottom ends of the fitting lines. (That a convineing Fig. 1 results makes no difference to the crudeness of eqn. (4).

With all points plotted and the bottom of the diagram fixed by formula, the rough picture of fitting lines like those of Fig. 1 emerged, with parallel lines in the supercritical zone $\left(\mathrm{F}_{b}\right.$ greater than $\left.g=32.2 \mathrm{ft} / \mathrm{sec}^{2}\right)$, and somewhat illdefined ones (particularly for some materials) in the subcritical. The supercritical parallelism was marked enough to cause a strong belief that it was not accidental. If it were not, then there must be a different law in the subcritical, for the higher parallel lines could not, by any stretch of imagination, indicate $\mathrm{F} b_{0}$ values approximating actuality. Normal hydraulic happenings suggest that the change of laws would occur through a transition, but, with scattered data, it seemed best to show a discontinuous change. The change was fixed at critical velocity, viz $F_{b}=g$, partly because it is a wellknown hydraulic figure and partly because the records seemed to indicate that the change from dunes to sheet flow occurred in its vicinity. It was done in spite of the fact that the initial rapid check, described at the beginning of this section, showed the change to occur at a much higher $\mathrm{F}_{b}$ value; regarding the data for that check GILBER'T says "the position of the: critical points being estimated by Mr. Murpiry when the details of the experiments were fresh in mind ". Presumably this conflict between memory and records could have been explained at the time, and may have resulted from Mr. Murpry keeping on the safe side according to some rational system; now it can only be recorded and given weight if some inconsistency of results arises. At this stage Fig. 1 consisted of the lower starting points, undetermined curves in the subcritical zone, discontinuities at critical velocity, and parallel lines in the supercritical zone.

The next stage was to test whether the lines could be collapsed into one discontinuous one, by using non-dimensional variables, and made to yield a definite family equation from which Fig. 1 could be redrawn with definite fitting lines. The simple hypothesis was made that, at least in the subcritical phase :

$$
\mathrm{F}_{b}=\mathbf{F}_{b 0}\left[1+f_{n}(\mathrm{C})\right]
$$

where in $(\mathrm{C})$ would have to vanish with $\mathrm{C}$ to suit the physical fact that $F_{b 0}$ exists at vanishing $\mathrm{C}$ and is given approximately by eqn (4). So the obvious procedure was to plot :

$$
\left(\mathrm{F}--\mathrm{F}_{b 0}\right) / \mathrm{F}_{b 0} \text {, }
$$

which may be called the "relative bed-factor excess", against $\mathrm{C}$. This was done on double log 
paper for dunes only and showed a relationship quite definitely although with scatter of points too great to prevent a clear decision on whether a straight line or gentle curve would be the better fit. (Luckily for the next step in analysis there was very little doubt about the charges at critical velocity, used to check parallelism in the supercritical zone.) There were no points with charge less than 8 or greater than 500 , and the points were most prolific for charges above about 80. The decision was made to fit the points with :

$$
\mathrm{F}_{b}=1.9 \mathrm{D}_{m^{0.5}}(1+0.068 \mathrm{C})
$$

although a non-linear law of $\mathrm{C}^{5 / 0}$ could have been used just as well. The subcritical lines of Fig. 1 are plotted from eqn (6) and terminated at :

$$
F_{b}=g=32.2 f t / \mathrm{sec}^{2} \text {. }
$$

The final step was to plot, for supercritical points only,

$$
\mathrm{F}_{{ }_{b}}-g \text { against } \mathrm{C}-\mathrm{C}_{c}
$$

where $\mathrm{C}_{0}$ was the charge found from eqn (6) by putting $\mathrm{F}_{b}=g$. This test for parallelism of the supercritical lines was made on ordinary squared paper, on which $\mathrm{C}-\mathrm{C}_{\mathrm{c}}$ ranged from about minus 80 to plus 1,700 with the points most prolific below 800 . The fitting line was definitely indicated to be straight and through the origin, with equation :

$$
\mathrm{F}_{b}-g=0.056\left(\mathrm{C}-\mathrm{C}_{\mathrm{c}}\right)
$$

The supercritical lines of Fig. 1 are plotted from eqn. (7).

The final conclusions were that bed-factor retains its utility as a criterion of "bed-factor " right up to, but not necessarily including, charges that cause antidunes, and that Fig. 1 was a reasonable formalised representation of the functional relation connecting bed-factor with material size and charge, pending the acquisition of better data.

\section{INVESTIGATION OF T'TEM II}

The test of equation ( $3 a$ ) was conducted by calculating :

$$
\mathrm{K}=\mathrm{V}^{2} / g d \mathrm{~S} \text { divided by }(\mathrm{V} b / \mathrm{y})^{0.25} \text {, }
$$

which would be 3.63 for field canals on which regime theory was based, and plotting the result on ordinary squared paper against $C$. In the subcritical range a convincing straight line was obtained passing through a value of 3.63 at $\mathrm{C}=$ zero. Beyond the critical the points went wild but seemed to be oscillating about a constant value for some materials and to actually drop for others. As in investigation $i$. antidune points were omitted. The equation of the line in the subcritical zone was :

$$
\mathrm{V}^{2} / g d \mathrm{~S}=3.63(1+\mathrm{C} / 400)(\mathrm{V} b / v) 0.25
$$

This was anticipated in writing equation (3) in this article. Because of the unwieldiness of the plot on ordinary squared paper it was altered, for publication, to Fig. 2 which tests on double log paper whether:

$\mathrm{V}^{2} / g d \mathrm{~S}$ divided by $(\mathrm{V} b / \mathrm{y})^{0.25}$ really scatters about $(1+\mathrm{C} / 400)$. The Figure is carried into the supereritical zone for material $\mathrm{D}$, which gives the most consistent results, to show how the equation ( 3 a) breaks down suddenly when eritical flow conditions are reached.

The final conclusions were:

(a) The regime slope formula constant of 3.63 is really a function of charge and can be amended, for the suberitical zone and lor uniform sediment within the range of investigation, to :

$$
3.63(1+\mathrm{C} / 400)
$$

and probably for any sediment that would retain its identity during flow to:

$$
3.63(1+a \mathrm{C})
$$

where " $a$ " will have to be determined from observation.

(b) Present data (probably due to inaccuracies of slope measurements in flumes) cannot give a rela fion for supercritical flow, but show clearly that the subcritical one would not apply to supercritical conditions.

The fact that the regime slope formula needed nothing more than a multiplier to make it fit the range of large charges of flume data seemed such strong confirmation of its dynamical correctness of form that Fig. 3 was prepared, partly for design purposes, and partly as a record of how the generalisation had occurred. Original regime theory gave the Nrr line from the Reynolds Number range of about $10^{6}$ to $10^{8}$; flume data gave the charge lines from the $10^{5}$ to $10^{6}$ range. The charge lines are extended into the regime theory range on the grounds that the improved formula has not lost its original identity or possible dynamical meanings. The reader should be cautious in extend ing this Figure to practice as the spacing of the $\mathrm{C}$ lines above NrL will be different from that shown if natural sands are used (10); so far as data show at present the position of the NiL line is not affected appreciably by whether sand is uniform or natural.

\section{Second analysis (11)}

The preliminary analysis had concentrated on GILbErT data, which concerned sands and fine gravels made "uniform" artificially. It had omitted antidune points, several groups of sheet flow points that seemed unlikely to add useful information to the plots (this was a labor-saving action believed to be random), and a few points whose status as to dunes or sheet flow the records had left vague. So the decision was made to reanalyse the whole of the GILBERT data, with a fresh mind, and to seek for data from other sources, particularly data for natural river-bed materials.

The attempt to find non-GubBert data drew attention to how little factual work has been done since he wrote in 1914, and to the superiority of his methods, industry and presentation. Subsequent work from which the authors tried to obtain iniormation all suffers from one or more of the following 
defects; (i) flumes so short that depths had not reached uniformity, (ii) flumes so short that slopes could not be accepted as pertaining to the short reach of uniform flow, (iii) conditions not allowed to become steasly, (iv) unnatural materials used so that suspended and bed-loads occurred together but were measured as one, $(v)$ conditions of initiation of bed-load movement mixed with those after initiation, (vi) very small range, (vii) large range with seatter of data uncompensated by the number of observations, (viii) original data inaccessible. So, no data other than Gilbert's were available or acceptable for the uniform materials, and only data by G. L. STruub [16] and the U.S. Corps of Engineers [17] for natural river-bed materials.

$i$. Bed-Factor, Gilbert. To avoid bias in favor of the idea of a discontinuity of law at critical velocity, as in Fig. 1, the decision was made to plot. sheets or relative bed-factor excess, $\left(\mathbf{F}_{b}-\mathbf{F}_{b 0}\right) / \mathbf{F}_{b, 1}$, against charge for every Glubert point. Fig. 4 is one of these plots for grade D material. Fig. 5 shows the fitting lines for all plots plus those for natural materials.

That Fig. 1 shows a discontinuity and Fig. 5 shows none is not remarkable. First, in plotting highly scattered data, even for one independent variable, a variety of fitting curves based on different physical idieas will be found to lie within the limits of statistical significance. Second, the various mathematical devices for finding equations to fitting curves are analogous to different optical devices - ienses, distorting mirrors, etc. - for looking at things; they can easily straighten curves and hide discontinuities. Specifically, suppose that the subcritical lines of Fig. 1 were replaced by the $5 / 6$ power curves that were stated to be equally good [see below eqn (6)]; they would not look much different. Then suppose these new lines replotted to the coordinates of Fig. 5. They would become straight lines of slope $5 / 6$ and conform exactly to the A - D lines, except perhaps for a little displacement without change of slope; they would not conform to the $\mathrm{E}-\mathrm{H}$ lines, but that would hardly be noticed because those lines have practically no coverage by points in the subcritical zone. This all draws attention to the facts that the fine materials lack points in the antidune zone, the coarse lack them very badly in the dune zone, and all lack them in the zone of low charge where they are needed so badly to start the curves properly.

In the analysis for fixing the exact lines of Fig. 1 a Fig. 5 was drawn for the subcritical zone, but the points were fitted with one line instead of the several of Fig. 5. Which method is correct can be proved only by further experiments. The statistical existence of parallel lines may be due to the peculiar way in which GILBERT rendered material "uniform ", by separating through sieves of adjacent size. If settlement velocity rather than particle size is the dynamical criterion for transport then the finer materials, for which settlement velocity varies approximately as the square of size, would be dispersed, effectively, three times as much as the coarser materials whose settlement velocity varies as the square root of particle size, assuming the sieves go in a fixed ratio; the little evidence that exists suggests that dispersion raises lines.

If $b / d$ and $\mathrm{D} / d$ were factors relevant to the definition of bed-factor in the range of study, scatter of points prevented their separation.

Final conclusions for uniform sediments were :

(a) bed-factor is a valid criterion of bed-load for all phases of bed-load movement.

(b) Bed factor is a function of particle size and of charge.

(c) Available data possess inadecuacies that prevent exact definition of the functional relation between bed-factor, particle size and charge. It may be relatively simple, but discontinuous, as shown by Fig. 1, or somewhat more complex but continuous as represented by Fig. 5 . The lines of those figures are equally reasonable within the limits of statistical significance.

ii. Bed-Factor, Nateral SANDS. A preliminary investigation on the particle size distribution in samples of alleged natural riverbed sand confirmed What the sedimentary petrologists seem to have known for a long time and the engineers have recently started to rediscover [14], viz that such sands follow the normal law of error in terms of logarithm of particle size, with the frequency measured in terms of weight. An interesting quantitative discovery was made, that :

Standard deviation of log. size $=2,6 \times(\text { settlement velocity of median })^{5 / 12}$

That dispersion increases with the mean size of natural materials is well known. The form of the dispersion equation for sands does not seem to have any particular physical significance; it applies from velocities of 0.8 to $35 \mathrm{~cm} / \mathrm{sec}$. Gravels generally plotted, on logarithmic probability paper, as bent straight lines with a noticeable family resemblance. It was decided that the dividing line between gravels and sands might be fixed somewhat roughly at $1 \mathrm{~mm}$; no doubt it is actually associated with the point of change of settlement velocity law to propor. tionality to root $D$.

The decision from the investigation was that median grain size was a fair criterion for sands, but a fair average size for gravels could not be stated. Also it was decided that USWES sands that fell outside certain somewhat arbitrary limits of agreement with the log. probability distribution should be rejected from regime analysis.

The STraus data were treated exactly like those of Gilusert and plotted on Fig. 5. The accepted USWES ones had to receive special treatment as they were for runs of the order of one hour each and experience has shown that regime in such experiments is approached asymptotically, so that times of the order of a day or two are necessary for final depths to occur with a given charge and discharge. The authors considered that as the experiments must have appeared to have reached a 
limit, the depths would probably be somewhere from $85 \%$ to $90 \%$ of ultimate. Therefore, merely for finding the functional relation with $\mathrm{C}$, it would be fairly accurate to calculate relative bed-factor excess in terms of the $F_{b 0}$ found by extrapolating a plot of observed $F_{b}$ against $C$, thereby cancelling errors of depth.j This action was taken and the results are on Fig. 5. Practically all points were for dunes.

The following conclusions seemed fair, for natural river sands in the dune phase (and perhaps, by extrapolation, beyond it) :

(a) Bed-factor is a valid criterion of bed-load.

(b) Size of bed-material may be represented by median particle size.

(c) Bed-factor is a function of particle size and charge.

(d) The functional form is probably the same as for uniform materials except perhaps for D since the factor of grain dispersion must be included in $\mathrm{D}$; the constants are probably different.

iii. Regime Slope Equation, Uniform and Natural SANDS. The original test of the preliminary analysis was carried ont for all data. That is :

$$
\mathrm{K}=\mathrm{V}^{2} / g d \mathrm{~S} \text { divided by }(\mathrm{V} b / \mathrm{v})^{0.25}
$$

was plotted against $\mathrm{C}$ on ordinary squared paper. In the preliminary analysis the range of $\mathrm{C}$, being for dunes, was quite small and the plot showed a linear relation. In the second analysis the inclusion of antidune data caused the dune zone to shrink into the left-hand corner of the sheet so that the plotted points seemed meaningless; see Fig. 6 . However, when the subcritical line of equation $(3 a)$ was drawn on the plots of all materials, and the best was made of the terribly scattered points of the supercritical zone, Fig. 7 emerged as what refined large-scale experiments might be expected to show. It may be noted that the only natural sand data are of Straub; those of the Corps of Engineers did not record slopes.

The conclusions were (a) For dunes, the regime slope equation is :

$$
\mathrm{V}^{2} / g d \mathrm{~S}=3.63(1+a \mathrm{C})(\mathrm{V} b / v)^{0.25}
$$

where $a$ is about $1 / 400$ for uniform materials and is probably different for natural river-bed ones. (b) When dunes pass into sheet flow a transition occurs from equation (3), and the ultimate state seems to be one in which $(1+\alpha C)$ takes a fixed value dependent on the size of the material.

(c) The scatter of data in the supercritical phases prevents any statement on numerical values to expect for constants there.

\section{Dimensional analysis}

Dimensional analysis, as applied to the relatively simple problems of rigid boundary hydraulics, uses only some of the possible alternative non-dimensional groups. For mobile boundary work there is merit in first obtaining the formulas that regime theory combined with plottings of actual data show to be reasonable, and then considering the extent to which dimerisional analysis shows that they might have to be generalized.

Adopting this method, we can argue that, if a flume of width $b$ is set up in a world of gravitational field $g$, and into it is run a discharge $Q$ of a fluid of density $o_{f}$ and kinematic viscosity $v$, carrying a charge $\mathrm{C}$ of uniform material of diameter $\mathrm{D}$ and density $\mathrm{o}_{s}$ then it is observed that a regime will establish itself with depth $d$, energy gradient $g \mathrm{~S}$, a distribution of shear stress $\tau$ over the boundary, and a mean velocity $V$. In mathematical shorthand:

$$
d, g \mathrm{~S}, \tau, \mathrm{V}=\mathrm{fns} \text {. of }\left(b, \mathrm{C}, \mathrm{D}, \mathrm{Q}, g, v, \mathrm{e}_{s}, \mathrm{p}_{f}\right)
$$

From our physical viewpoint we elect to call the four variables on the left "dependent" and the eight on the right "independent"; the mathematician is just as willing to put any four on the left and the remaining eight on the right. As the three basic units of mass, length', and time are contained in all the variables the preceding equations can be replaced by the same number of equations amongst new variables made of nondimensional groups, and then the number of independent variables will be eight minus three equals five. In physical fact there nay be less than five, but, so far as dimensional requirements are concerned there cannot possibly be more than five. The reader may verify that the following equations satisfy dimensional requirements completely :

$$
\begin{aligned}
\mathrm{V}^{2} / g d & =\mathrm{fn} . \text { of }\left(\mathrm{V} d / \nu, \mathrm{C}, b / d, \sqrt[3]{g \vee} . \mathrm{D} / \nu, \mathrm{o}_{s} / \mathrm{o}_{f}\right) \\
& =\mathrm{fn} . \text { of }\left(\mathrm{V} d / \nu, \mathrm{C}, b / d, \mathrm{D} / d, \mathrm{\rho}_{s} / \mathrm{e}_{f}\right) \\
\mathrm{V}^{2} / g d \mathrm{~S} & =\mathrm{fn} . \text { of }\left(\mathrm{V} b / \nu, \mathrm{C}, b / d, \mathrm{~V}^{2} / g b, \mathrm{o}_{s} / \mathrm{e}_{f}\right)
\end{aligned}
$$

(To verify, check that the left-hand side is a nondimensional function of one or more dependent variables, that the right contains five groups, that each group contains a term absent from the others, and that all independent variables have been used.)

Equations (X), (Y) are generalised possibilities (in the dimensional sense, though they may be overgeneralised for physical reality) for equations (5) - (7); equation (Z) corresponds to equation (3). Apparently all the equations from analysis may depend on relative density, and there is no doubt the bed-factor ones actually do, and the slope one may; in field practice this quantity does not vary, nor does it in the experiments that gave the data, so we may drop it from further argument. Apparently also bed-factor and slope equations may depend on $b / d$ and there seems no physical doubt that they must, but the factor is not important enough to show up within its range in the data (Table I). An interesting point is that $\mathrm{Vd} / \nu$ may be relevant in the bed-factor formulas; that it did not prove to be so in the analysis is not surprising when one considers the irrelevance of its equivalent in a wide range of rough rigid pipe experiments, but there is a warning that it might become important at low values outside the range of the data. Equation (Y) shows that the expression for $\mathrm{F}_{l, 0}$ might change from dependence on $D$ to dependence on $D / d$ for large $D / d$, and raises the question whether 
this dependence exists in the supereritical data but escaped notice because of the small variations of the small $d$ in that range; it has been admitted already that the data prove next to nothing about the exact form of dependence on $D$ even in the subcritical range. The term $\mathrm{V}^{2} / g b$ in equation (Z) may be physically irrelevant if there is foundation for the belief that $\mathrm{V} * / g d$ is, in some way, a measure of the balancing of the uplift due to turbulence generated at the bed against the weight of the particles that form the bed; there is no side material to uplift as there is on the bed. Finally, the peculiar term $(v / g)^{1 / 3} \mathrm{D} / \nu$ probably indicates that settlement velocity would be a better term, dynamically, than particle size.

On the whole there is nothing to indicate inadequacy of the formulas for the range of data they represent, but there are some good clues to why they are adequate and where they may cease to be adecluate outside the range of data or on the edges of the range.

\section{Cooperative research}

To remove the deficiencies shown in the preceding pages will require cooperative research by agencies including those with large funds and large discharges of water. (Some organisations can divert several hundreds of cusecs from canals, and back again, with negligible trouble.) The immediate practical objective is to find, for natural channel bed materials, and for clean water at standard temperature, the relationship of both bed-factor and the regime slope equation parameter $K$ to bedmaterial size and bed-load charge through the three phases of movement (dune, sheet, and antidune), but particularly in the first phase. The basic investigations leading to full attainment of the objective could proceed, to some degree, simultaneously. Thus, the investigation of the minimum length of flume required for attainment of uniform conditions and measurable slope need not precede work in flumes or channels that are obviously of adequate length; on the other hand experiments cannot be conducied with natural gravel till extensive field analyses (probably calling for help from road administrations, gravel pit operators, and geomorphologists) have shown the law of mechanical constitution of active natural river-bed gravels. An international conference, or several cooperating national ones [18], could plan a tentative division of labor. Principal heads of research would be :

$i$. Length of flume and time of observation to obtain (a) steady conditions of bed-load transport and $(b)$ accurate measurement of uniform slope.

ii. Law mechanical constitution of active natural river-bed gravels.

iii. Determination of the relationship of bedfactor and regime-slope parameter $\mathrm{K}$ to bed-material size and charge, for bed-load transport by clean water at standard temperature, using discharges up to $500 \mathrm{cfs}$ covering dune, sheet and antidune phases, and paying special attention to the previouslyneglected conditions of :

(a) Naturally-graded sands of all sizes.

(b) Material sizes finer than $0.3 \mathrm{~mm}$ down to $0.1 \mathrm{~mm}$.

(c) Material sizes from $7 \mathrm{~mm}$ up to about $40 \mathrm{lb}$.

(d) Naturally-graded gravels.

(e) Satisfactorily uniform sizes less than $0.4 \mathrm{~mm}$.

(f) Clarges from vanishingly small amounts to about 30 parts per hundred thousand by weight.

(y) Transitions between phases.

(h) Appreciable size of bed-material relative to depth as with supercritical flow over gravel.

iv. Efrect of fluid viscosity on relationships.

v. Effect of suspended load on apparent viscosity.

$v i$. Behavior of specially uniform materials, e.g. glass or plastic dodecahedrons, not subject to the differences of shape and density that occur in natural materials.

vii. Effect of relative density of load material and fluid.

All data should be published in original.

\section{APPENDIX I. - NOMENCLATURE}

All units in fi-sec units unless stated otherwise

$b=$ Breadth of channel.

$\mathrm{C}=$ Charge as ratio of weight of sediment per second divided by weight of water per second, and reduced to thousandths of a percent.

$\mathrm{C}_{\mathrm{v}}=$ Charge at critical velocity.

$\mathrm{K}=$ Generalised form of nondimensional constant in King equation.

$d=$ Depth of flow.

$\mathrm{D}=$ Diameter of bed-material particles.

$\mathrm{D}_{m}=$ Diameter of bed-material particles measured in millimeters.

$F_{b}=$ The bed-factor, $\mathrm{V}^{2} / d$.

$\mathrm{F}_{b 0}=$ The bed-factor when $\mathrm{C}$ is vanishinglhy small.

$g=$ Acceleration of gravity.

$\nu=$ Kinematic viscosity.

$a=$ A constant number.

$S=$ Channel slope for conditions where watersurface slope is presumed the same.

$\mathrm{V}=$ Mean velocity of flow.

$x=\mathrm{A}$ constant number.

The letters A-Y are used, as GilberT and Murpfy used them, to identify the sand grades used.

$f n=$ means " any unspecified function of ".

$\mathrm{K}=\left(\mathrm{V}^{2} / g d \mathrm{~S}\right) \div(\mathrm{V} b / \mathrm{v})^{0.25}$.

$\rho=$ mass density.

$\tau=$ shear stress intensity. 
TABLE I : COMPARATIVE RANGES OF DATA

\begin{tabular}{|c|c|c|c|c|}
\hline & Canals & GrubERT: & MEYER-PETER & Enstein \\
\hline 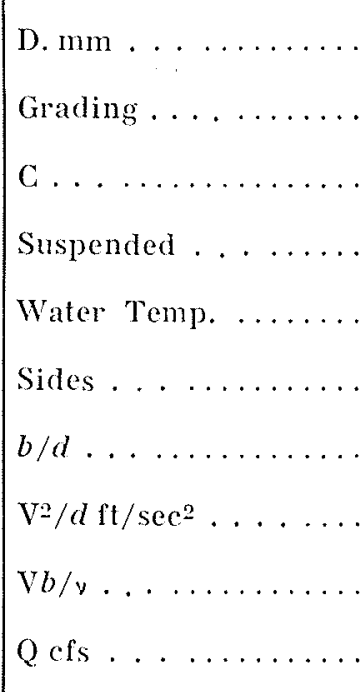 & $\begin{array}{l}0.10-0.60 \\
\text { L.og. Prob. } \\
0-2 \text { or } 3 ? \\
0-1 \% \\
50-86^{\circ} \mathrm{F} \\
\text { Clay, smooth } \\
4-30 \\
0.5-1.5 \\
106-10^{\mathrm{s}} \\
1-10,000\end{array}$ & $\begin{array}{l}0.3-7.0 \\
\text { Uniform } \\
0-3,000 \\
\text { Nil } \\
\text { Prob. } 55^{\circ} \mathrm{F} . \\
\text { Wood or glass, smooth } \\
1-25 \\
1-150 \\
10^{5}-10^{\circ} \\
0.1-9.0\end{array}$ & 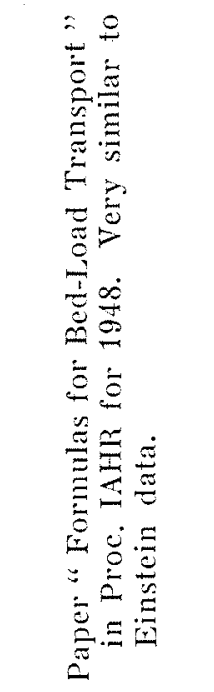 & 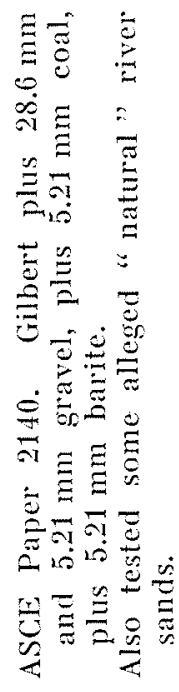 \\
\hline Bed Phase......... & Dunes & \multicolumn{3}{|c|}{ Dunes, Sheet and Antidunes } \\
\hline
\end{tabular}

REFERENCES, - BIBLIOGRAPHIE

1. Hydraulies of Sediment-Bearing Canals and Rivers. T. Blench. U.S.A. Copyright 1951. (From author.)

2. Civil Engineering Reference Book. BUTEERworths. 1951. Chapter on "Canals, Channels and Rivers"

3. Regime Theory for Self-Formed Sediment-Bearing Channels. T. Buench. Trans. ASCE 1942. Paper 2499.

4. The Transportation of Debris by Running Water. Grove Earl Griberr. Based on Experiments made with the Assistance of Edward Charles Murray. U.S. Geological Survey Paper 86. 1914.

5. Régime Theory Equations applied to a Tidal Estuary. T. Blexch. Proc. International Association for hydraulic Research, Joint Convention with ASCE, Minneapolis, September 1953.

6. The Hydraulic Geometry of Stream Channels and Some Physiographic Implications. Luna B. LeoPoLd and Thomas MabDogk Jr. U.S.G.S. Professional Paper 252. 1953.

7. Formulas for Bed-Load Transport. E. MEYEn-PETrn and R. Muller. Proc. International Association for Hydraulic Research. 1948.

8. Formulas for the Transportation of Bed Load. H. A. EINstein. Assoc. M.ASCE. Trans. ASCE. 1942. Paper 2140.

9. The Bed-Load Function for Sediment Transportation in Open Channel Flows. H. A. Ersstern. U.S. Dept. of Agriculture, Soil Conservation Service, Tech. Bulletin 1026. September 1950.
10. Practical Regime Theory Design of Artificial Channels with Self-Adjustable Boundaries, T. BLENCH. ASCE Separate No. of

11. Thesis on Analysis of Existing Bed-Load Data, by R. B. ERs, as part requirement for degree of M. Sc. from University of Alberta 1955 .

12. Regime Formulas for Bed-Load Transport. 'T. BLENCH. Proc. IAHR 1955.

13. Constitution of the Research Committee of the Central Board of Irrigation with Resolutions... Central Board of Irrigation, Simla, India. 1940.

14. Normal Size Listribution Found in Samples of RiverBed Sand. Civil Engineering, Feb. 1952. Tr. BLENCH.

15. Proceedings Central Board of Irrigation, India. 1943. Paper by C. Kisci. Available U.S.B.R. Library, Denver.)

16. Terminal Report on Transportation Characteristics, Missouri River Sediment. Lorenz G. Straub. M.R.D. Sediment Series, Corps of Engineers, No. 4, April 1954

17. Studies of Riner Bed Materials and their Monement.. U.S. Waterways Experiment Station, Prof. Paper 17 of 1955 .

18. Notes from the Fontana Conference on Sediment Transport, oct. 11-15, 1954. Sponsored by the University of Tennessee, The Sedimentation Committee of the Hydraulies Division of the ASCE, and the National Science Foundation of the U.S.A. 